\title{
A trans-acting long non-coding RNA represses flowering in Arabidopsis
}

Yu Jin ${ }^{1}$, Maxim Ivanov ${ }^{1}$, Anna Nelson Dittrich ${ }^{2}$, Andrew D. L. Nelson² and Sebastian Marquardt ${ }^{*}$

${ }^{*}$ Correspondence: sebastian.marquardt@plen.ku.dk

${ }^{1}$ Department of Plant and Environmental Sciences, Copenhagen Plant Science Centre, University of Copenhagen, Frederiksberg, Denmark

Boyce Thompson Institute, Cornell University, Ithaca, NY, USA

\section{Keywords}

IncRNA, FLAIL, trans-acting, chromatin binding, flowering regulation, ChIRP-seq

\section{Abstract}

Eukaryotic genomes give rise to thousands of long non-coding RNAs (IncRNAs), yet the purpose of IncRNAs remains largely enigmatic. Functional characterization of IncRNAs is challenging due to multiple orthogonal hypothesis for molecular activities of IncRNA loci. Here, we identified a flowering associated intergenic IncRNA (FLAIL) that represses flowering in Arabidopsis. An allelic series of flail loss-of-function mutants generated by CRISPR/Cas9 and T-DNA mutagenesis showed an early flowering phenotype. Gene expression analyses in flail mutants revealed differentially expressed genes linked to the regulation of flowering. A genomic rescue fragment of FLAIL introduced in flail mutants complemented gene expression defects and early flowering, consistent with trans-acting effects of the FLAIL RNA. Knock-down of FLAIL RNA levels using the artificial microRNA approach revealed an early flowering phenotype shared with genomic mutations, indicating a trans-acting role of FLAIL RNA in the repression of flowering time. Genome-wide detection of FLAIL-DNA interactions by ChIRP-seq suggested that FLAIL may directly bind genomic regions. FLAIL bound to genes involved in regulation of flowering that were differentially expressed in flail, consistent with the interpretation of $F L A I L$ as a trans-acting IncRNA directly shaping gene expression. Our findings highlight $F L A I L$ as a trans-acting IncRNA that affects flowering in Arabidopsis, likely through mediating transcriptional regulation of genes directly bound by FLAIL.

\section{Background}

The purpose of DNA sequences that do not encode proteins represents an open question in the biology of genomes. It is now clear that RNA polymerase II (RNAPII) converts non-coding DNA into non-coding RNA genome-wide [1]. Long non-coding RNAs (IncRNAs) are key products of ubiquitous RNAPII transcription in the non-coding genome [2]. Non-coding DNA engaged in IncRNA production may function through various molecular mechanisms, ranging from roles as DNA elements, RNAs, the act of transcription and small peptides [3,4]. This wide range of possible cellular roles affects strategies to elucidate their functions experimentally [5]. Functional characterization of DNA elements benefits from a high-quality annotation of IncRNAs in genomes [6]. Integration of several orthogonal 
transcriptomic data offers an opportunity to inform on the precise location of various IncRNA subtypes and alternative IncRNA isoforms from a single locus [6]. Experimental avenues to abolish specific IncRNA isoforms may trigger the generation of alternative isoforms that may partially substitute for functions, calling for a multi-facetted functional characterization of IncRNA loci $[7,8]$. The resulting RNA molecules, but also the act of transcription generating IncRNA can regulate the expression of neighboring genes [9]. The act of transcription from an upstream IncRNA locus may trigger gene activation of the downstream, or gene repression, for instance by transcriptional interference [9]. Ribosome profiling (Ribo-seq) may suggest small open reading frames (sORFs) of the IncRNA loci [3]. Notably, IncRNA association with ribosomes may indicate either ribosome-coupled RNA degradation or translation [3]. Collectively, the broad range of candidate hypotheses by which IncRNA loci may play functional roles call for multiple approaches to distinguish alternative molecular mechanisms [10].

LncRNAs may regulate nearby genes in cis or distant genes in trans [11-13]. Compared to cis-acting IncRNAs, relatively fewer functions of trans-acting IncRNAs have been clarified [4]. A key experiment to distinguish between cis-acting and trans-acting mechanisms is to test phenotypic complementation of IncRNA loss-of-function mutants by IncRNA expression from a different genomic region [4]. Transacting IncRNAs regulate distant genes via different mechanisms [11], for example, through chromatin targeting of IncRNAs to fine tune chromatin architecture resulting in an altered transcriptional output [14]. Nevertheless, functional characterization of trans-acting IncRNAs remains a key knowledge gap to understand the regulatory contributions of the non-coding genome.

In plants, an increasing number of IncRNA loci have been implicated in the regulation of flowering time [15-20]. Flowering time represents a developmental transition of plants that is key for reproductive success. Genetic and environmental factors, for example, altered internal secondary metabolites (e.g. lignin), extended cold periods (i.e. vernalization) or day length (i.e. photoperiod), help plants to align flowering with favorable conditions [21-23]. Vernalization-induced flowering associates with several IncRNAs such as COOLAIR, COLDAIR, ANTISENSE LONG (ASL), and COLDWRAP that in cis repress gene expression of FLOWERING LOCUS C (FLC), a key flowering repressor at different stages of vernalization [24-27]. The contribution of trans-acting IncRNAs to the regulation of flowering time is currently unclear.

Here, we characterized the IncRNA locus FLAIL in Arabidopsis that gives rise to several RNA isoforms. Genomic mutations and strand-specific RNA repression provided evidence that FLAIL sense IncRNA repressed flowering. Genetic complementation data supported a trans-acting role of $F L A I L$ in the regulation of flowering genes. FLAIL RNA bound the chromatin of flowering-related target genes that were differentially expressed in flail mutants, arguing for direct effects of $F L A I L$ in flowering gene regulation. In summary, our data suggest flowering regulation through effects on gene expression by chromatin association of the trans-acting RNA FLAIL. 


\section{Results}

\section{Characterization of the FLAIL locus}

FLAIL was annotated as a IncRNA in the TAIR [28] and GreeNC [29] databases and mapped as a single exon to chromosome 2 in Arabidopsis (Fig. 1A). Consistently, Nanopore RNA-seq data of chromatin-associated RNAs provided no evidence for splicing at the FLAIL locus [30] (Fig. S1A). Additionally, plaNET-seq used for genome-wide profiling of nascent RNA polymerase II (RNAPII) transcription [31], identified both sense and antisense isoforms at the FLAIL locus (Fig. 1A). We obtained additional information by examining RNA $5^{\prime}$-end mapping by TSS-seq [32] and simultaneous RNA 5'- and 3'-end mapping by TIF-seq [33] in the mutant of the nuclear exosome component HUA ENHANCER2 (i.e. hen2-2). These data confirmed that the FLAIL locus was transcribed on both strands, since sense and antisense FLAIL transcripts were detected. Moreover, RNA isoforms derived from FLAIL were $5^{\prime}$-end capped, $3^{\prime}$-end polyadenylated and degraded by the nuclear exosome in Arabidopsis (Fig. 1A). To assess the protein-coding potential of FLAIL RNAs, we used the Coding Potential Calculator (CPC2) [34] and Coding-NonCoding Identifying Tool (CNIT) [35]. These analyses revealed poor coding potential of the IncRNA FLAIL, similar to other well-known ncRNAs (18sRNA, U6, ELENA1, SVALKA) [36, 37], but much lower than the protein coding potential of UBIQUITIN(UBQ) mRNA (Fig. 1B). Finally, an analysis of translation start sites in FLAIL RNAs suggested poor protein coding potential, well below the 0.5 threshold predicted for protein start codon as determined by the NetStart software package [38] (Fig. S2). Nevertheless, Ribo-seq [39, 40] data indicated ribosome association of FLAIL RNAs. This ribosome association could be consistent with translation of two sORFs with $~ 9$ amino acids, or ribosome-mediated RNA degradation (Fig. S1B) [39]. In summary, the FLAIL locus harbors both sense and antisense IncRNA isoforms.

Even though IncRNAs show relatively poor sequence conservation [41], trans-acting IncRNAs may show signatures of conservation across species [42]. We identified a match in the genome of Camelina sativa on chromosome 17 (Fig. S3A). Camelina, like Arabidopsis, is in the Brassicaceae and last shared a common ancestor with Arabidopsis 18 million years ago [43]. Reciprocal blast searches narrowed down a microhomology region between the sense FLAIL 3 '-end in Arabidopsis and a non-coding DNA region near the Camelina gene Csa17g011930. In turn this region in Camelina is syntenic to a genomic region in Arabidopsis surrounding the At1G08480 locus (Fig. S3A). Indeed, we successfully detected the existence of genomic FLAIL-like non-coding DNA by PCR amplification (Fig. S3C). We next performed RT-PCR targeting the FLAIL-like non-coding DNA region to examine RNA expression in Camelina sativa leaves and seedlings (Fig. S3B-C). Our data are consistent with the expression of RNA from the FLAIL-like non-coding DNA region in Camelina sativa, even though we detected noticeably weaker expression compared to Arabidopsis FLAIL expression in equivalent experimental conditions. In conclusion, our comparative genomic analysis identified a candidate FLAIL-like non-coding DNA region in Brassicaceaes with signatures of RNA expression. 


\section{FLAIL characterizes a trans-acting IncRNA repressing flowering}

113 To address the function of FLAIL in Arabidopsis, we used CRISPR/Cas9 technology with paired

114 sgRNAs to generate two different flail knockout mutants with deletion fragments of 229 bp (flail1) [44], and 343 bp (flail2) (Fig. S4A-E). We also obtained a mutant line SAIL_645_C03 (flail3) carrying a TDNA insertion at the $3^{\prime}$-end of sense FLAIL locus (Fig. S4A, F). All three mutants reduced the expression of sense and antisense FLAIL transcripts as detected by RT-qPCR, suggesting that they reduced the bioavailability of FLAIL isoforms (Fig. 1C-D). All three flail mutants flowered earlier than wild type (Fig. 1E-F). Our genetic data thus revealed a link between non-coding transcription at the FLAIL locus and flowering time.

To test which transcript of FLAIL was linked to the observed phenotype of the flail mutant we performed a complementation test. We transformed a DNA fragment encoding either sense or antisense $F L A I L$ driven by the corresponding native promoter into the flail3 mutant background with GUS driven by $35 S$ promoter as a control (Fig. 2A). Importantly, the constructs carried the NOS terminator that largely abolished initiation of antisense transcription [8, 37, 45]. We selected flail3 for complementation because FLAIL disruption by T-DNA insertion at the 3 '-end left potential sORF regions at the $5^{\prime}$-end intact (Fig. S4A). We selected two representative homozygous single locus insertion lines for each transformed construct (flail3 pFLAIL:gFLAIL18/88 and flail3 pasFLAIL:gasFLAIL18/39) that expressed sense and antisense FLAIL at levels slightly higher than or similar to wild type (Fig. 2B-C). However, only exogenous expression of sense FLAIL rescued the early flowering phenotype of the flail3 mutant (Fig. 2D-E), suggesting that the early flowering of the flail mutant was caused by the disruption of the sense FLAIL transcript isoform. In Arabidopsis, transgene insertion is non-targeted. We thus reasoned that complementation argued for the capability of sense FLAIL to act from a different genomic location, presumably as a trans-acting IncRNA. This hypothesis predicts that knock-down of sense FLAIL RNA should result in equivalent effects as genomic mutations. To test this hypothesis, we employed strand-specific RNA repression using artificial microRNAs (amiRNAs) [46]. We generated two amiRNA targets (amiR-FLAIL-11 and amiRFLAIL-12) (Additional file1: Fig. S5A), both exhibiting strongly reduced sense FLAIL transcription (Additional file1: Fig. S5B) and similar effects on flowering as genomic mutations (Additional file1: Fig. S5C-D). In conclusion, our experimental data indicate that the sense FLAIL IncRNA represses flowering through a trans-acting mechanism.

FLAIL IncRNA binding to chromatin regions promotes the expression of selected flowering repressors

Early flowering in Arabidopsis may be associated with altered expression of flowering-related genes. To gain insight into the molecular basis of FLAIL-mediated regulation of flowering time, we determined 
the transcriptional profiles of two-week old seedlings for wild type, flail3, and flail3 pFLAIL:gFLAIL with at least two independent replicates using stranded RNA-seq. Since the early flowering time effect in flail3 could be rescued by $p F L A I L: g F L A I L$, we reasoned that this experimental setup may be suitable to identify gene expression changes directly correlated with sense $F L A I L$ expression. Compared to wild type, 1221 differentially expressed genes (DEGs) were called by DESeq2 with at least two-fold change (adjusted $p$ value < 0.05; Table S1), with 419 up-regulated and 802 down-regulated genes in flail3 mutants. Almost half of these transcriptional differences in flail3 reverted to wild-type expression level by exogenous expression of the FLAIL sense RNA into flail3 mutants, including the FLAIL IncRNA itself (Fig. S6A and Fig. S7F, Table S2). We next focused on functional annotations of genes associated with the process of flowering [47]. Among the DEGs in flail3, we identified twenty genes linked to flowering (Fig. S6A). Expression of most of them were fully (eight) or partially (five) rescued by the expression of $p F L A I L: g F L A I L$ (Fig. 3A-E, Fig. 4E-H, Fig. S6B, S7A-E and S9B, D). In conclusion, our transcriptomic data indicate potential targets for the trans-acting IncRNA FLAIL. The reversible effect on flowering gene expression upon re-introduction of $p F L A I L: g F L A I L$ argued for a direct effect of the FLAIL IncRNA. To identify where FLAIL bound chromatin regions, we performed chromatin isolation by RNA purification (ChIRP) of endogenous FLAIL followed by DNA-seq (FLAIL ChIRP-seq). We targeted sense FLAIL by two non-overlapping antisense oligonucleotide pools (Even and Odd probes, Fig. 4A bottom) tiled along the entire FLAIL transcript sequence compared to an oligonucleotide pool against the LUCIFERASE (LUC) mRNA as control that is not expressed in our strains. We efficiently captured the endogenous FLAIL RNA from chromatin compared to the unrelated $U B Q$ mRNA (Fig. 4B). Moreover, LUC-specific probes did not enrich FLAIL, suggesting the specificity of RNA affinity purification in our assay conditions (Fig. 4B). After isolating DNA fragments associated with the FLAIL-containing complex, we assessed the genome-wide occupancy of FLAIL at high resolution by ChIRP-seq, followed by peak calling using CCAT3.0 [48]. Only peaks that occurred at target genes from both even and odd probe pools, but not from two or more independent experiments of Luc pools were considered significantly enriched (Fig. S8A). This analysis strategy allowed us to identify 210 target genes of FLAIL (Table S3). We observed a strong enrichment for the FLAIL locus by both ChIRP-seq (Fig. 4A, top) and ChIRP-qPCR (Fig. 4C). We noted that the FLAIL locus represented the only locus where we could expect binding, thus validating our identification strategy of genomic FLAIL binding. We analyzed the overlap between genes which were differentially expressed in the RNA-seq experiment and those genes that showed statistically significant FLAIL binding by ChIRP-seq (Fig. 4F). We identified twelve genes matching both criteria. At these targets, chromatin binding by $F L A I L$ was linked to corresponding changes of gene expression, arguing for direct effects of $F L A I L$ binding on gene expression. Four of these genes were functionally connected to flowering: CIRCADIAN 1 (CIR1), LACCASE 8 (LAC8), PECTIN LYASE-LIKE 25 (PLL25) and genes were transcriptionally down-regulated in flail3 (Fig. 4D-E and 4G-H, Fig. S9A-D, Table S1). 
However, the complementation construct failed to restore NMT3 expression, arguing against a direct effect here (Fig. S9A-B, Table S2). A detailed examination revealed that PLL25 regulates flower morphology rather than flowering time [49], arguing against effects on flowering time through misregulation of this gene [50,51]. On the other hand, cir1 [52] and lac8 [53] mutants displayed early flowering phenotypes. These data suggest repression of $C I R 1$ and LAC8 as candidate molecular hypothesis to explain the early flowering time phenotype in flail $[52,53]$. In conclusion, our data suggest that $F L A I L$ sense RNA represses flowering through inhibiting expression of the direct $F L A I L$ targets $C I R 1$ and $L A C 8$, consistent with a model where gene expression changes regulating flowering are mediated by interactions of the trans-acting IncRNA FLAIL with the genome (Fig. 4I).

\section{Discussion}

\section{FLAIL functions in flowering time}

In this study, we report repression of flowering time by the trans-acting IncRNA FLAIL. While many IncRNAs affect flowering regulation, the molecular mechanisms remain largely unclear [15-20]. Previously characterized IncRNAs including COOLAIR, COLDAIR, ASL, COLDWRAP, and MAS are transcribed from the flowering genes FLC or MAF4 to locally affect their coding gene expression in cis $[20,24,27,54,55]$. We note that the $F L A I L 3^{\prime}$-end resides approximately 130 bp upstream of the 5' UTR region of the PORCUPINE (PCP, also called Sm protein E1, SME1) gene, and pcp/sme1 mutants flower early $[56,57]$. We failed to find evidence for the hypothesis that FLAIL may affect flowering through $P C P$ by a cis-acting mechanism. FLAIL neither regulates $P C P$ expression nor shows overlapping effects on gene expression (Table S1 and S5-7, Fig. S10A-D).

In contrast, our work identifies a trans-acting FLAIL that represses flowering and binds multiple genomic loci. We thus favor the interpretation that FLAIL binding to other genomic regions explains the phenotypic effects of the mutants. For instance, FLAIL promotes the expression of the MYB family transcription factor $C I R 1$, which in turn broadly contributes to gene expression. This may also explain why some genes are FLAIL-regulated but lack chromatin interactions with FLAIL. CIR1 is a circadian clock gene, induced by light and involved in a regulatory feedback loop that controls a subset of the circadian outputs [52]. Our GO analysis supports that a subset of DEGs are connected to the response to red or far red light that contains among other key flowering genes such as PHYTOCHROME INTERACTING FACTOR 4 (PIF4) and CONSTANS (CO) (Fig. S10B). FLAIL also binds the chromatin region of LAC8. LAC8 is a laccase family member that mainly modulates phenylpropanoid pathway for lignin biosynthesis [53, 58]. Similar to flail, lac8 mutants flower early [53]. While intermediates in this pathway $[59,60]$ or dysregulation of lignin-related genes $[21,22,61]$ could promote flowering in plants, the molecular connections of reduced LAC8 expression to effects on flowering time will require further investigation. Nevertheless, our observations of reduced expression 
of $L A C 8$ and $C I R 1$ in flail, combined with restored expression upon re-introduction of $F L A I L$, and direct FLAIL binding to $L A C 8$ and CIR1 chromatin, suggested that early flowering in flail may result from combined direct and indirect effects of $L A C 8$ and $C I R 1$ repression. The range of plausible candidate mechanisms by which FLAIL may promote gene expression includes targeting of chromatin modifying complexes to activate gene expression as shown for the mammalian IncRNA HOTTIP [62], or effects on pre-mRNA RNA processing to stimulate mRNA expression as indicated for the plant IncRNA ASCO [63-65]. While our results clarify key aspects of functional units of the non-coding genome, it will remain an exciting future research endeavor to elucidate how sense FLAIL mediates the activation of floral repressor genes at the molecular level.

\section{Identification of the functional FLAIL RNA isoform of the FLAIL locus}

A challenge in the field of IncRNA biology is the functional characterization of IncRNA loci. Our functional dissection of $F L A I L$ illustrates these challenges, yet reveals a compelling example for a trans-acting IncRNA. Like many other loci, FLAIL generates multiple transcript isoforms, including cryptic transcript isoforms that would be missed by most standard transcriptomic approaches. DNA regulatory elements embedded in IncRNA loci and the act of IncRNA transcription may regulate the expression of neighboring genes (cis-acting) $[66,67]$. However, disruption of the FLAIL locus had no significant impact on gene expression in a surrounding $\sim 150 \mathrm{~kb}$ genomic window (from upstream AT2G18560 to downstream AT2G18940). These observations argue against the hypothesis that the FLAIL locus acts as a cis-acting RNA or DNA element. In contrast, we found genetic evidence that the FLAIL sense IncRNA functions as a trans-acting RNA. First, exogenous expression of FLAIL sense RNA in vivo rescues the early flowering phenotype as well as the expression of flowering genes in flail3. The rescue is specific for the FLAIL IncRNA encoded by the sense strand. Second, amiRNA mutants that specifically knock-down FLAIL sense RNA levels without effect on the DNA also show early flowering. Collectively, these data indicate that the role of the FLAIL locus in the repression of flowering is executed by a trans-acting IncRNA derived from the sense strand. We assessed the protein-coding potential of FLAIL using a series of software tools that give low scores, arguing against a protein encoded by FLAIL. Nevertheless, Ribo-seq identifies ribosome association of FLAIL, consistent with two small ORFs with $\sim 9$ amino acids (Fig. S1B) [39]. Even though we found no evidence for peptide production in proteomics data, sORFs embedded in IncRNAs may encode for functional peptides $[68,69]$. In our allelic series of flail mutants only flail1 mutates the potential sORFs, while flail2 and flail3 keep the DNA sequences encoding the potential sORFs intact. The range of genetic mutations thus argue against the contribution of potential sORFs peptides to flowering, since the early flowering phenotype is similar in all three mutant backgrounds. Moreover, the microhomology with Camelina mapped to the 3'-region of FLAIL where we identified no ribosome association. While future experimental research in Camelina sativa would be needed to 
examine functional conservation in flowering, the microhomology of FLAIL in other Brassicaceae is consistent with functional RNA domains that are a characteristic of conserved trans-acting IncRNAs.

\section{Conclusions}

In summary, this work highlights the contribution of IncRNAs to fine tune the complex developmental transition to initiate flowering. Regulation for key developmental decisions by IncRNA-based mechanisms may confer specific organismal advantages, yet detecting the functional roles of the noncoding genome may also be facilitated in the developmental context. RNA-based regulation of plant reproductive development through trans-acting IncRNA promises future possibilities for plant breeding research to improve Brassicaceae crop quality and resilience.

\section{Methods}

\section{Plant materials and growth conditions}

Table S8 provides a complete overview of the plant materials used in this study. Seeds of flail3 (SMA2648, Table S8) were obtained from the Nottingham Arabidopsis Stock Centre and genotyping was performed using primers Mlo37/1788/1789 (Additional file 2 Table S9). A. thaliana plants were grown in a growth chamber with a long day photoperiod (16-h light/8-h dark) at $20^{\circ} \mathrm{C}$ at a photo flux density of approximately $100 \mu \mathrm{mol} / \mathrm{m}^{2} / \mathrm{sec}$ in the chamber. For seedling treatments, seeds were surface-sterilized and placed on $1 / 2 \mathrm{MS}+1 \%$ sucrose plates at $4{ }^{\circ} \mathrm{C}$ in darkness for 3 days prior to germination. Then, plates were placed in the growth chamber in control conditions at $20^{\circ} \mathrm{C}$ and sampled.

\section{CRISPR/Cas9-directed flail mutants}

Construction of a dual sgRNA-directed flail1 (goi, [44]) knockout SMA3677 (Table S8) was performed by CRISPR/Cas9 using the described protocol [44]. Similarly, to generate the CRISPR mutant flail2 (SMA3678, Table S8), plasmid pHEE2E-TRI (SMC528) harboring two sets of gBlocks including gBlock1 (a U6-26 promoter, a 19 bp target sequence 1, a sgRNA scaffold, a terminator) and gBlock2 (a U6-29 promoter, a 19 bp target sequence 2, a sgRNA scaffold, a terminator) served as a template to amplify the middle border (Bsal-overhang1-protospacer1-scaffold-terminator-U6-29 promoterprotospacer2-overhang2-Bsal) using primer paris Mlo1757 \& Mlo1758 (Table S9). Second, both pKIR1.1 plasmid (SMC529) and the middle border were digested by Aarl and Bsal, respectively, then the middle border was integrated into the linearized pKIR1.1 backbone to generate pKIR1.1-dualsgRNA2 SMC575 (Table S10) for plant transformation. Third, after transformation using Agrobacterium tumefaciens strain SMA111 (Table S11), the T1 generation plants for successful TDNA insertion events were selected by identifying seeds with red fluorescence, then seeds from individual T1-genotyped plants were harvested and grown. Finally, T2 mutants that did not contain 
the CRISPR/Cas9 construct were identified by picking up T2 seeds without red fluorescence. PCR products were amplified from Cas9-free T2 plants using oligonucleotides Mlo2478/2479 (Table S9) flanking the deletion site for Sanger sequencing.

\section{Complementation assay}

Complementation constructs were generated using SMC431. pFLAIL:FLAIL and pasFLAIL:asFLAIL were amplified from genomic wild type DNA using primers MLO1746/1747 and MLO1759/1761, respectively (Table S9). The resulting PCR products were inserted into pENTR-D-Topo by topo cloning to generate entry vectors SMC542 and SMC541 (Table S10). The entry vectors were used in a LR reaction with SMC431 to generate expression vector SMC546 (containing pFLAIL:gFLAIL construct) and SMC545 (containing pasFLAIL:gasFLAIL construct) (Table S10). The complementation constructs together with the control vector 35S:GUS (SMC377) were then transformed into GV3101 to get strains SMA112-114 (Table S11), followed by transformation into the flail3 mutant. Seeds from transformed Arabidopsis plants were screened for T-DNA integration by hygromycin resistance. Multiple independent single-locus insertions were identified by segregation analysis and homozygous T3 transgenic plants SMA4477 for flail3 35S:GUS, SMA4462 for flail3 pFLAIL:gFLAIL18, SMA4464 for flail3 pFLAIL:gFLAIL88, SMA4467 for flail3 pasFLAIL:gasFLAIL18, SMA4468 for flail3 pasFLAIL:gasFLAIL39 were used for the complementation assay (Table S8).

\section{Cloning of amiRNA}

To construct amiR-FLAILs, we designed two artificial miRNA sequences in the miR319a backbone using Web MicroRNA Designer (WMD3) software to generate four oligonucleotide sequences (I to IV) (Mlo1774-1781, Table S9), which were used to engineer the amiRNA into the endogenous miR319a precursor by site-directed mutagenesis. The amiRNA containing precursor was generated by overlapping PCR using SMC532 as a template following the protocol described in the WMD3 publication [46]. The fragment containing the amiRNA sequence was then introduced into pENTR-DTopo by topo cloning to generate entry vectors (SMC547 for amiR-FLAIL11-Topo and SMC548 for amiR-FLAIL12-Topo) (Table S10). The entry vectors were used in a LR reaction with SMC531 to generate expression vector SMC558 (containing amiR-FLAIL11 construct) and SMC559 (containing amiR-FLAIL12 construct). Plant transformation was performed using Agrobacterium strains SMA115117 (Table S11). Finally, homozygous T3 transgenic plants SMA4469, SMA4471, and SMA4480 (Table S8) were used in this study.

\section{Gene expression analysis by PCR/RT-(q)PCR}

For the comparative expression analysis with $C$. sativa, genomic DNA was extracted from wild type A. thaliana and $C$. sativa seedlings ( 1.5 weeks) and mature leaves ( $\sim 4$ weeks) using the DNeasy Plant Mini kit (Qiagen \#69104) and diluted to $5 \mathrm{ng} / \mu \mathrm{l}$. Total RNA was extracted from the same tissues 
using the RNeasy Plant Mini kit (Qiagen \#74904) with RNase-free DNase set (Qiagen \#79254). Reverse transcription (RT) was performed on $1 \mu \mathrm{g}$ of DNase-treated total RNA using the iScript cDNA synthesis kit (Bio-Rad \#1708890), with the same amount of total RNA diluted in water as negative controls for RT-PCR. Primers (Table S9) were designed to amplify AthaFLAIL (AT2G18735), CsatFLAIL-like (C. sativa, Ensembl v51 Chr17 3432317-3432814), and GAPDH as an amplification control. Two reverse primers were designed to target different portions of AthaFLAIL and CsatFLAILlike. The GAPDH primers amplify both AthaGAPDH and CsatGAPDH. PCR was performed with templates consisting of either $20 \mathrm{ng}$ of genomic DNA, $50 \mathrm{ng}$ of total RNA, or $50 \mathrm{ng}$ of total RNA that had been reverse transcribed. 35 cycles of amplification were performed for all template types. PCR products were visualized after agarose gel electrophoresis using the Bio-Rad Chemi-doc imaging platform.

For reverse transcription quantitative real-time PCR, total RNA was extracted from two-week old Arabidopsis seedlings using an RNeasy Plant Mini Kit (Qiagen, Germany). DNA in the isolated RNA were digested with TURBO DNase (Thermo Fisher Scientific, USA). Purified RNA was subsequently reverse transcribed into $\mathrm{cDNA}$ with iScript ${ }^{\mathrm{TM}} \mathrm{cDNA}$ Synthesis Kit (Bio-Rad, USA) following manufacturer's instructions. For real-time PCR analysis, the resulting cDNA was diluted ten-fold and used as a template in a PCR reaction with GoTaq qPCR Master mix (Promega, USA) and run on a CFX384 Touch instrument (Bio-Rad, USA) with an initial denaturation at $95^{\circ} \mathrm{C}$ for 2 minutes, followed by 40 cycles at $95^{\circ} \mathrm{C}$ for 15 seconds, $60^{\circ} \mathrm{C}$ for 1 minute. Primer efficiencies were evaluated on a standard curve generated using a 10-fold dilution series of the sample over four dilution points. Relative expression was calculated and normalized to the internal reference gene UBQ. All primers (Table S9) did not show any evidence for non-specific products in the melting curve analysis.

\section{Chromatin isolation by RNA purification (ChIRP)}

ChIRP was performed as previously described with some modifications [70]. Probe design: The antisense oligonucleotide probes were designed against the full-length FLAIL sequence using the online probe designer at www.singlemoleculefish.com [71]. The probes were biotinylated at the $3^{\prime}$ end. To assess the specificity of the target capture by the oligonucleotides, the FLAIL probes were divided into two pools, Odd (Mlo2885) and Even (Mlo2886). All the experiments were carried out using both pools independently. Sixteen biotinylated oligonucleotide probes complementary to the LUCIFERASE transcript were pooled (Mlo2887) as negative control (Table S9). RNA immunoprecipitation: Two-week old seedlings were crosslinked in $3 \%$ formaldehyde solution by vacuum infiltration for 25 minutes and then quenched by the addition of $0.125 \mathrm{M}$ glycine at room temperature for 5 minutes. After washing and drying, crosslinked seedlings $(2.5 \mathrm{~g})$ were ground to a fine power in liquid nitrogen and suspended in $10 \mathrm{ml} \mathrm{Honda} \mathrm{Buffer} \mathrm{(0.44} \mathrm{M} \mathrm{Sucrose,} \mathrm{1.25 \%} \mathrm{Ficoll,}$ 
mM PMSF, $1 \times$ Cocktail, and $2 \mathrm{U} / \mathrm{ml}$ RNase inhibitor). The samples were put on ice and mixed gently at $4{ }^{\circ} \mathrm{C}$ until the solution became homogenous, then filtered through two layers of Miracloth, centrifuged at $4000 \mathrm{rpm}$ for 15 minutes at $4{ }^{\circ} \mathrm{C}$. Pellets were resuspended in $1.5 \mathrm{ml}$ Honda buffer and centrifuged at $4000 \mathrm{rpm}$ for 15 minutes at $4^{\circ} \mathrm{C}$. Resuspending and centrifugation were repeated until the pellet was no longer green, typically two more times ( 15 minutes). Pellets were suspended in $1600 \mu \mathrm{l}$ nuclear lysis buffer $(50 \mathrm{mM}$ Tris- $\mathrm{HCl}, \mathrm{pH}$ 7.5, 2 mM MgCl $2,1 \%$ SDS, $0.1 \mathrm{mM}$ PMSF, $10 \mathrm{mM}$ EDTA, $1 \mathrm{mM}$ DTT, $1 \times$ Cocktail, $0.1 \mathrm{U} / \mu$ l RNase Inhibitor) and sonicated at M setting 5 minutes (30 seconds on and 30 seconds off) $\times 3$ times by sonicator (QSONICA Q700, USA) until DNA was fragmented into $200-500 \mathrm{bp}$ pieces. After centrifugation at $12,000 \mathrm{~g}$ at $4{ }^{\circ} \mathrm{C}$ for 10 minutes, the supernatant (around $1.5 \mathrm{ml}$ ) was diluted with 2 volumes of pre-heated hybridization buffer $(750 \mathrm{mM}$ $\mathrm{NaCl}, 1 \%$ SDS, $50 \mathrm{mM}$ Tris, pH 7.5, $1 \mathrm{mM}$ EDTA, 15\% formamide, and $1 \times$ protease inhibitor). The clear mixture was divided into 4 equal aliquots (IP-RNA and IP-DNA for both Even and Odd probes). Biotinylated DNA probe $(2 \mu \mathrm{l}$ of $100 \mathrm{pmol} / \mu \mathrm{l})$ was added to each aliquot and incubated at $37^{\circ} \mathrm{C}$ for 4 hours with gentle mixing. Then $50 \mu \mathrm{l}$ of well-washed Streptavidin $\mathrm{C} 1$ magnetic beads were added to each sample and incubated at $37^{\circ} \mathrm{C}$ for 30 minutes. Captured beads were washed three times with high salt wash buffer ( $2 \times$ SSC, $0.5 \%$ SDS, $1 \mathrm{mM} \mathrm{DTT}$, and $1 \mathrm{mM} \mathrm{PMSF}$ ) and three times with low salt wash buffer $(0.1 \times$ SSC, $0.5 \%$ SDS, $1 \mathrm{mM} \mathrm{DTT}$. and $1 \mathrm{mM} \mathrm{PMSF})$.

RNA isolation: Beads were resuspended in $200 \mu \mathrm{RNA}$ elution buffer $(100 \mathrm{mM} \mathrm{NaCl}, 50 \mathrm{mM}$ Tris- $\mathrm{HCl}$ with $\mathrm{pH}$ 7.5, $1 \mathrm{mM}$ EDTA, 1\% SDS) and boiled for 15 minutes. RNA samples were treated with Proteinase $\mathrm{K}(1 \mathrm{mg} / \mathrm{ml})$ at $65^{\circ} \mathrm{C}$ for $1 \mathrm{~h}$ while shaking. RNA was extracted using TRIzol method, and then treated with DNase (Qiagen). RNA was used for RT-qPCR analysis to confirm RNA retrieval.

DNA isolation: DNA was eluted with elution buffer $(200 \mathrm{mM} \mathrm{NaCl}, 50 \mathrm{mM} \mathrm{NaHCO} 3,1 \% \mathrm{SDS}, 10 \%$ SDS, $0.1 \mathrm{U} / \mu \mathrm{l}$ RNase $\mathrm{H}$ ). After Proteinase $\mathrm{K}$ treatment at $45^{\circ} \mathrm{C}$ for $1 \mathrm{~h}$, DNA was extracted using ChIP DNA Clean \& Concentrator (Zymo Research) and used for subsequent qPCR analysis or highthroughput sequencing.

\section{Comparative Genomics:}

FLAIL sequence similarities were initially identified through reciprocal best BLAST against eleven disparate Brassicaceae genomes in CoGe BLAST [72] with an e-value of $10^{-5}$. As no hits were identified in a species more distantly related than Camelina sativa, comparative analysis was restricted to Arabidopsis and Camelina. Genomic regions displaying sequence similarity to the Arabidopsis FLAIL locus in the Arabidopsis thaliana (TAIR10) and Camelina sativa (Ensembl v2.0) were identified using CoGe BLAST, with regions of microsynteny identified using GEvo [73]. Nucleotide sequence for syntenic regions were extracted and imported into Geneious Prime v2021.2.2 [74]. Multiple sequence alignments were used to design primers that specifically amplified a portion of FLAIL and the FLAIL-like intergenic region in Camelina. 


\section{Statistical analysis}

The number of rosette leaves were measured with ImageJ software [75]). Statistical analysis was performed using the R software [76] or GraphPad Prism 9 [77]. For comparisons, data were evaluated using Student's t-test for significance of differences: * means $p$ value $<0.05$, ${ }^{* *}$ means $p$ value $<0.01$.

\section{Bioinformatics}

All the supporting code for bioinformatics analysis is repository available at FLAIL_2021 from GitHub (https://github.com/Yu-Jin-KU) [78].

\section{Sequencing analysis}

RNA-seq libraries were prepared using NEXTFLEX® Rapid Directional RNA-seq Library Prep Kit (NOVA-5138-08) and ChIRP-seq libraries were constructed using the ChIP-seq Library Prep Kit (NOVA-5143-02 with NEXTflex® ChIP-seq Barcodes-24) following the manufacturer's protocol and quantified on Agilent 2100 Bioanalyzer. Both RNA-seq and ChIRP-seq libraries were pooled into one flow cell (NextSeq 500/550 High Output Kit v2.5) for sequencing in PE mode (2*75 bp) on Illumina Nextseq 500.

Previously published RNA-seq datasets for wild type and PCP (SME1) deficient plants were downloaded from European Nucleotide Archive (accession number PRJEB24412) and from Gene Expression Omnibus (accession number GSE116964). The raw reads were quality controlled by the FastQC software [79], adapter trimmed by Trimmomatic v0.39 [80] in paired-end mode and then aligned to TAIR10 genome assembly by STAR v2.7.8a [81] in Local mode. Aligned reads with MAPQ below 10 were removed by Samtools v1.1.2 [82]. BAM files were converted to unstranded Bedgraph files using BEDtools genomecov v2.30.0 [78]. The code was detailed in the RNA-seq.sh script on the GitHub page mentioned above. Differentially expressed genes (DEGs) were called using the DESeq2 tool [83]. Expression fold change values were log2 transformed to identify genes with statistically significant differential expression using the following criteria: $\mid \log 2$ fold change $>1$ and adjusted $p$ value $<0.05$. GO enrichment analysis was done by Metascape [47].

ChIRP-seq reads were quality controlled by the FastQC software [79], adapter trimmed using Trim Galore v0.6.7 [84] and mapped to the TAIR10 genome using STAR v2.7.8a [81]. The converted BAM files were sorted and filtered for $M A P Q \geq 5$. After removing PCR duplicates by Samtools v1.1.2 [82], peaks of FLAIL occupancy were called with CCAT3.0 [48]. By using CHIPseeker [85] and TxDb.Athaliana.BioMart.plantsmart28 [86] packages in R, peak annotation was performed with the definition for a promoter being $300 \mathrm{bp}$ around the TSS. Alignment statistics were provided in Table S3. Visualization and analysis of genome-wide enrichment profiles were done with IGV genome browser [87]. 


\section{Abbreviations}

amiRNAs: artificial microRNAs

440 ChIRP: chromatin isolation by RNA purification;

441 CIR1: CIRCADIAN 1;

442 CNIT: Coding-NonCoding Identifying Tool;

443 CO: CONSTANS;

444 CPC2: Coding Potential Calculator;

445 DEGs: differentially expressed genes;

446 FLAIL: flowering associated intergenic IncRNA;

447 FLC: FLOWERING LOCUS C;

448 LAC8: LACCASE 8;

449 IncRNAs: long non-coding RNAs;

450 LUC: Luciferase;

451 NMT3: PHOSPHOETHANOLAMINE N-METHYLTRANSFERASE 3;

452 sORFs: small open reading frames;

453 PCP: PORCUPINE;

454 PIF4: PHYTOCHROME INTERACTING FACTOR 4

455 plaNET-seq: plant Native Elongating Transcripts sequencing

456 PLL25: PECTIN LYASE-LIKE 25;

457 RNAPII: RNA polymerase II;

458 SAMs: shoot apical meristems;

459 SME: Sm protein E1;

460 TIF-seq: Transcript Isoform sequencing;

461 TSS-seq: Transcription Start Site sequencing;

462 UBQ: UBIQUITIN

463 WMD: Web MicroRNA Designer

\section{Figure legends}

467 Fig. 1 Phenotypes of FLAIL knock-down plants. A Genome browser screenshot of plaNET-seq, TSS468 seq and TIF-seq at the FLAIL genomic region in Col-0 and hen2-2 mutant. Sense (+) and antisense 469 (-) strands were shown in red and dark blue, respectively. Grey bar and light blue bar indicated primer 470 locations of RT-qPCR for sense FLAIL (FLAIL) and antisense FLAIL (asFLAIL), respectively. B 471 Coding potential of the transcript in the genomic region of FLAIL and asFLAIL and reference 472 transcripts including non-coding RNAs (18sRNA, U6, ELENA1, SVALKA) and coding gene UBQ 473 according to the CNIT and CPC2 algorithm. C-D Detection of FLAIL and asFLAIL gene expression in 
Col-0 and flail mutants by RT-qPCR. Transcript levels were normalized to UBQ expression levels. Yaxis showed relative values compared to the expression level of Col-0. Bars represented average \pm s.e.m ( $\mathrm{n}=3$ independent $14-\mathrm{d}$ seedling pools). ${ }^{*}, p$ value $<0.05$ and ${ }^{* *}, p$ value $<0.01$ by Student's t-test compared to Col-0. E Morphological phenotypes of 4-week-old plants of Col-0, flail mutants at $20^{\circ} \mathrm{C}$ under a 16-h light/8-h dark growth condition. Scale bar: $2 \mathrm{~cm}$. F Violin graph showed number of rosette leaves after appearance of the first flower bud in Col-0. Data represented the mean of six independent experiments. Boxes spanned the first to third quartile, bold black lines indicated median value for each group and whiskers represented the minimum and maximum values. ${ }^{*}, p$ value $<0.05$ was indicated by Student's t-test compared to Col-0.

Fig. 2 Sense-FLAIL RNA is functional for flowering. A Schematic representations of the T-DNA constructs containing the native promoter of sense-FLAIL (native-Pro) fused to the sense-FLAIL DNA region with NOS as a terminator or the native promoter of anti-sense FLAIL (anti-native-Pro) fused to the anti-sense FLAIL DNA region or a negative control with the 35 S promoter (35S) fused to GUS reporter were transformed into flail3 Arabidopsis plants. B-C Detection of FLAIL and asFLAIL genes expression in Col-0, flail3 mutant and complemented lines expressing pFLAIL:gFLAIL and pasFLAIL:gasFLAIL by RT-qPCR. Transcript levels were normalized to $U B Q$ expression levels. $Y$ axis showed relative values compared to the expression level of Col-0. Error bars represented s.e.m ( $\mathrm{n}=3$ independent $14-\mathrm{d}$ seedling pools). ${ }^{*}, p$ value $<0.05$ and ${ }^{* *}, p$ value $<0.01$ by Student's t-test compared to Col-0. D Representative morphological phenotypes of 4-week-old plants of Col-0, flail3 mutant, transgenic lines at $20^{\circ} \mathrm{C}$ under a 16-h light/8-h dark growth condition. Scale bar: $2 \mathrm{~cm}$. E Violin graph showed number of rosette leaves after appearance of the first flower bud in Col-0. Data represented the mean of six independent experiments. Boxes spanned the first to third quartile, bold black lines indicated median value for each group and whiskers represented the minimum and maximum values. *, $p$ value $<0.05$ was indicated by Student's t-test compared to Col- 0 .

Fig. 3 FLAIL regulates flowering related genes. A Heatmap and hierarchical clustering of top 70 differentially expressed genes (DEGs) in flail3 versus Col-0 that were best rescued in the flail3 pFLAIL:gFLAIL 18 complementation line. DEGs analyzed by DESeq2 with |log2 fold change| $>1$ and adjusted $p$ value $<0.05$ were considered significant differential expression. Three biological replicates for flail3, wild type, and two for flail3 pFLAIL:gFLAIL18. Flowering genes were highlighted in yellow bold. Samples clustered together on the basis of corresponding similar expression profiles. The color scale reflected the log 2-fold change in gene expression, ranging from down-regulated (blue) to upregulated (red). B-E Genome browser screenshots illustrating the expression of dysregulated flowering genes in flail3 were rescued in complementation line (top panel). Normalized read counts (TPM from RNA-seq) for differentially expressed flowering genes in WT, flail3, and flail3 pFLAIL:gFLAIL18 plants (bottom panel). Boxes spanned the first to third quartile, bold black lines 
511 indicated median value for each group and whiskers represented the minimum and maximum values.

512 All $p$ values were denoted by Students' $t$-test. Bar $=500$ bp.

514 Fig. 4 FLAIL affects flowering by chromatin binding of flowering genes. A Top, FLAIL bound the locus itself by ChIRP-seq from two independent Odd and Even probed chromatins. Luc probe was used as a control. Bottom, schematic representation of the antisense oligonucleotide probes that were biotinylated at the 3'-end with Odd (in red) and Even (in dark blue) against FLAIL sense RNA and Luc probe (in grey) against $L U C$ mRNA. B ChIRP-qPCR using probe pools FLAIL-asDNA (Odd and Even) retrieved $\sim 5 \%-10 \%$ of $F L A I L$ endogenous RNA and $<1 \%$ levels of $U B Q$. LUC-asDNA probes retrieved much lower levels of both RNAs as a control. C FLAIL DNA signal was identified in both Odd and Even probes. UBQ region showed much less binding signal in all probes as a negative control. Graphs in B \& C showed the mean \pm s.e.m. ( $n=3$ independent replicates). D, G Genome browser screenshots illustrating two of FLAIL bound targets CIR1 (D) and LAC8 (G) in RNA-seq (lane 1-3) and ChIRP-seq (lane 4-6). FLAIL binding peaks were called by CCAT3.0 with the cutoff FDR = genes in WT, flail3, and flail3 pFLAIL:gFLAIL18 plants (bottom panel). Boxes spanned the first to third quartile, bold black lines indicated median value for each group and whiskers represented the minimum and maximum values. F Venn diagram of genes targeted by FLAIL (ChIRP-seq) and genes overlapped with DEGs in flail3 and flowering related genes. I A model for the trans-acting FLAIL sense RNA regulated flowering. The FLAIL sense RNA binds chromatin regions of flowering genes to regulate expression levels of flowering related genes and thus affects flowering time. All $p$ values were denoted by Students' t-test.

\section{Supplementary information}

535 Fig. S1 Characterization of FLAIL locus. A Genome browser view of FLAIL splicing status in nanopore sequencing of Col-0. Transcription of sense FLAIL RNA and asFLAIL RNA were shown in red and dark blue, respectively and no isoform resulting from alternative splicing was observed. B FLAIL nucleotide sequences in black and two sORFs locations in blue.

Fig. S2 Assessment of FLAIL and UBQ for protein coding potential. Initiation codon translational analysis using NetStart for FLAIL and UBQ. The predicted initiation codons were depicted with the letter i, other instances of "ATG" by the letter "N" (non-start). The dots (".") were place holders for all

543 the other sequence elements. The scores were always in $[0.0,1.0]$; when greater than 0.5 , they represented a probable translation start. 
546 Fig. S3 Sequence and transcriptional conservation at the FLAIL and FLAIL-like loci in Arabidopsis

547 and Camelina. A Schematic depicting the conservation of the Arabidopsis (A.tha) FLAIL locus in

548 Camelina sativa (C.sat). Green boxes represent exons, with triangles representing direction of

549 transcription. The FLAIL locus is represented by a yellow box. Faded blue lines represent sequence

550 similarity between different loci. Dashed boxes at the FLAIL and Csa17g011930 loci represent regions

551 targeted for RT-PCR. B Amplification of A.thaFLAIL and C.satFLAIL-like, using RNA template (+/- RT,

552 lanes 1-20). NT indicates no template was added to the reaction. C Control amplifications: GAPDH 553 was amplified using RNA template (+/- RT, lanes 21-29), and A.thaFLAIL and C.satFLAIL-like were 554 amplified using genomic DNA template (lanes 30-41). L indicates leaf tissue, $S$ indicates seedling 555 tissue.

Fig. S4 FLAIL dual-sgRNA approach and T-DNA insertion genotyping. A Schematic representation of targeted gene FLAIL with locations of two Cas9-directed mutants flail1, flail2, and one T-DNA mutant flail3. Locations of dual-sgRNA target sites were shown in red arrow, primer pair F/R (Mlo2478/2479) were used for PCR testing deletion in flail1 and flail2, triangle indicated the putative position of T-DNA insertion at the FLAIL locus. Blue boxes indicated two sORFs. B Similar to the previous generation of flail1, FLAIL2 construct containing pKIR1.1 enabled red fluorescence selection of flail2 seeds from T1 plants after transformation by floral dipping. C Genotyping of individual T1 plant with red fluorescence, Leaf genomic DNA of T1 plants were PCR amplified. Expected size of PCR product for deletion was $343 \mathrm{bp}$ in flail2 line. flail2-32 in red box was selected for next generation. D In T2 selection, the null mutant flail2-32-93 without red fluorescence in seeds represented Cas9-free plants, indicated by red box in E. PAM was shown in blue, sgRNA protospacers were in red, and deleted bases were replaced by dots. E PCR analysis of T2 lines. The expected size of the wildtype FLAIL amplicon and the deletion size between Cas9 cut sites in flail2-32-93 were indicated. This inherited Cas9-null segregation line was used for data analysis in Fig. 1. F Genotyping of the T-DNA insertion mutant flail3. Gel sample order (from left to right): lane 1, marker; lane 2, WT; lane 3, flail3, with primer set, Mlo1788 + Mlo1789 + Mlo37; Results clearly showed that flail3 line was homozygous since WT was the only line giving WT band and flail3 line gave the only T-DNA insertion band.

Fig. S5 FLAIL IncRNA functions as a flowering repressor. A Sequences and structures of amiRNA duplexes and the target sites of amiR-FLAILs and amiR-FLAIL*s. Upper panel, schematic representation of transcribed sense FLAIL RNA. Lower panel, sequences of amiRNA duplexes, amiRNA (in red) target sites, and potential amiRNA* (in blue) on cognate sense FLAIL (in black). B Gene expression of sense FLAIL in amiR-FLAIL plants by RT-qPCR. Transcript levels were normalized to $U B Q$ expression levels. Two representative lines for amiRNA designed transgenic plants were selected. Y-axis showed relative values compared to the expression level of empty vector transformed plants. Grey bars depicted the relative positions of primers used for RT-qPCR analyses. 
Transcript levels were normalized to $U B Q$ expression levels. Error bars represented s.e.m $(n=3$ independent 14-day seedling pools). ${ }^{* *}, p$ value $<0.01$ analyzed by Student's t-test. C Morphological phenotypes of 4-week-old plants of Col-0 and two amiR-FLAIL plants under a 16-h light/8-h dark growth condition. Scale bar: $2 \mathrm{~cm}$. D Violin graph showed number of rosette leaves after appearance of the first flower bud in Col-0. Data represented the mean of six independent experiments. Boxes spanned the first to third quartile, bold black lines indicated median value for each group and whiskers represented the minimum and maximum values. ${ }^{* *}, p$ value $<0.01$ was indicated by Student's t-test compared to Col-0.

Fig. S6 Genome-wide effects of FLAIL on gene expression by RNA-seq. A Venn diagram of flowering genes overlapped with upregulated and downregulated genes in the flail 3 mutant and with unchanged genes in the flail3 pFLAIL:gFLAIL18 complementation plant. B Reproducibility of all genes shown in Fig. 3 from RNA-seq data was demonstrated by clustered heatmap of Pearson correlation coefficients over all independent replicates of RNA-seq in WT, flail3 mutant, and flail3 pFLAIL gFLAIL18 plants. Darker red denoted higher correlation and darker blue represented low reproducibility.

Fig. S7 FLAIL regulates flowering related genes in trans. A-F Genome browser screenshots illustrating the expression of dysregulated flowering genes in flail3 were most fully rescued in complementation line. Normalized read counts (TPM from RNA-seq) were used for differentially expressed flowering genes in WT, flail3, and flail3 pFLAIL:gFLAIL18 plants. Boxes spanned the first to third quartile, bold black lines indicated median value for each group and whiskers represented the minimum and maximum values. $p$ value was denoted by Students' t-test.

Fig. S8 Strategy to identify genome-wide binding profile of FLAIL analyzed by ChIRP-seq. A Illustration of FLAIL peaks called by CCAT3.0. Only peaks that occurred at target genes from both Even and Odd probed DNA, but not from two or more independent experiments of Luc pools were considered significant enrichment (Example 1), peaks that only occurred from either Even or Odd pools were not considered FLAIL targets (Example $2 \& 3$ ). B A pie chart that was generated by ChIPseeker represented distribution of FLAIL bound regions in the genome. A total of 210 FLAIL bound regions were annotated according to the genomic distribution and FLAIL was enriched predominantly in promoter regions.

Fig. S9 FLAIL binds chromatin regions of flowering genes for gene regulation. A, C Genome browser screenshots illustrating two more FLAIL bound flowering targets PLL25 and NMT3 in RNA-seq (lane 1-3) and ChIRP-seq (lane 4-6). Their expression that was downregulated in flail3 (lane 1) can be partially or fully rescued in complementation line (lane 2). Both Odd and Even probes identified chromatin binding regions of FLAIL in two more flowering genes NMT3 and PLL25 compared to LuC 
probes. FLAIL binding peaks were called by CCAT3.0. B, D Normalized read counts (TPM from RNAseq) for differentially expressed (DE) flowering genes in WT, flail3, and flail3 $p F L A I L: g F L A I L 18$ plants (bottom panel). Boxes spanned the first to third quartile, bold black lines indicated median value for each group and whiskers represented the minimum and maximum values. $p$ value was denoted by Students' t-test.

Fig. S10 FLAIL regulates flowering independent of PCP. A, B Venn diagram showing overlapping differentially expressed genes (up/down) sets between flail3 and pcp (also called sme1) mutants. C, D GO analysis of the DEGs in RNA-seq data of flail3 and pcp /sme1 mutants. Y-axis indicated the GO categories including biological process (BP), cellular component (CC) and molecular function (MF); X-axis showed -Log10 $p$ value with the cutoff 0.05 . Highly enriched GO terms of dysregulated mRNAs analyzed by the Metascape with 5 top enrichment scores.

Table S1. DEGs in flail3 mutant

Table S2. DEGs in flail3 pFLAIL gFLAIL18 plants

Table S3. FLAIL targets called by CCAT3.0

Table S4. Overlapping genes between DEGs in flail3 and FLAIL targets.

Table S5. Gene Ontology of DEGs in flail3

Table S6. Gene Ontology of DEGs in pcp (RNA-seq data from Capovilla et al with the ENA accession: PRJEB24412)

Table S7. Gene Ontology of DEGs in sme1 (RNA-seq data from Huertas et al with the GEO accession: GSE116964)

Table S8. Seeds

Table S9. Primer sequences

Table S10. Plasmids

Table S11. GV3101 strains

646

Declarations

648

649

Ethics approval and consent to participate

650

Not applicable

651

652

\section{Consent for publication}

653 Not applicable 
The RNA-seq and ChIRP-seq data were deposited at GEO with the number GSE186215 [88]. The reagents described in this study are available from the corresponding author.

658

\section{Competing interests}

660 The authors declare they have no competing interests.

661

\section{Funding}

663 S.M. acknowledges the funding from the Novo Nordisk Foundation (NNF150C0014202, 664 NNF19OC0057485), a Copenhagen Plant Science Centre Young Investigator Starting grant and 665 EMBO YIP. This project receives support from the European Research Council (ERC) under the 666 European Union's Horizon 2020 Research and Innovation Programme (StG2017-757411 to S.M.). 667 A.D.L.N. would like to acknowledge funding from NSF-IOS 1758532 and NSF-IOS 2023310.

\section{Authors' contributions}

670 Y.J. and S.M. conceived the study. Y.J. performed most experimental work, A.N.D. and A.D.L.N 671 contributed comparative genomics and expression analysis in S3. M.I. and Y.J. did the computational analysis. Y.J. and M.S. wrote the manuscript.

\section{Acknowledgements}

675 We thank Prof. Anders H. Lund and for kindly providing Luc probes. We thank Prof. Peter Brodersen 676 for sharing amiRNA vectors. We thank Dr. Pan Zhu and Dr. Marta Montes for assistance with ChIRP, 677 Prof. Egle Kudirkiene and Dr. Quentin Thomas for sequencing. We thank Jasmin Dilgen, Evangelia 678 Lakita, Ida Damholt Richardt, Lei Li for technical assistance, Jan Høstrup for excellent plant care, Dr. 679 Deyong Zhu and the members of Marquardt lab for discussions and manuscript feedback. 
681

682

683

684

685

686

687

688

689

690

691

692

693

694

695

696

697

698

699

700

701

702

703

704

705

706

707

708

709

710

711

712

713

\section{References}

1. Osman S, Cramer P. Structural Biology of RNA Polymerase II Transcription: 20 Years On. Annu Rev Cell Dev Bi. 2020;36:1-34.

2. Kapranov P, Cheng J, Dike S, Nix DA, Duttagupta R, Willingham AT, Stadler PF, Hertel J, Hackermuller J, Hofacker IL, et al. RNA maps reveal new RNA classes and a possible function for pervasive transcription. Science. 2007;316:1484-1488.

3. Choi SW, Kim HW, Nam JW. The small peptide world in long noncoding RNAs. Brief Bioinform. 2019;20:1853-1864.

4. Gil N, Ulitsky I. Regulation of gene expression by cis-acting long non-coding RNAs. Nature Reviews Genetics. 2020;21:102-117.

5. Goff LA, Rinn JL. Linking RNA biology to IncRNAs. Genome Res. 2015;25:1456-1465.

6. Ivanov M, Sandelin A, Marquardt S. TrancriptomeReconstructoR: data-driven annotation of complex transcriptomes. Bmc Bioinformatics. 2021;22:290.

7. Jin Y, Marquardt S. Winter fields antisense RNAs to kick off flowering. Gene Dev. 2021;35:785-786.

8. Zhao YS, Zhu P, Hepworth J, Bloomer R, Antoniou-Kourounioti RL, Doughty J, Heckmann A, Xu CY, Yang HC, Dean C. Natural temperature fluctuations promote COOLAIR regulation of FLC. Gene Dev. 2021;35:888-898.

9. Gowthaman U, Garcia-Pichardo D, Jin Y, Schwarz I, Marquardt S. DNA Processing in the Context of Noncoding Transcription. Trends Biochem Sci. 2020;45:1009-1021.

10. Chen L, Zhu QH, Kaufmann K. Long non-coding RNAs in plants: emerging modulators of gene activity in development and stress responses. Planta. 2020;252:92.

11. Kopp F, Mendell JT. Functional Classification and Experimental Dissection of Long Noncoding RNAs. Cell. 2018;172:393-407.

12. Ariel F, Lucero L, Christ A, Mammarella MF, Jegu T, Veluchamy A, Mariappan K, Latrasse D, Blein T, Liu C, et al. R-Loop Mediated trans Action of the APOLO Long Noncoding RNA. Mol Cell. 2020;77:10551065.

13. Moison M, Pacheco JM, Lucero L, Fonouni-Farde C, Rodriguez-Melo J, Mansilla N, Christ A, Bazin J, Benhamed M, Ibanez F, et al. The IncRNA APOLO interacts with the transcription factor WRKY42 to trigger root hair cell expansion in response to cold. Mol Plant. 2021;14:937-948.

14. Mishra K, Kanduri C. Understanding Long Noncoding RNA and Chromatin Interactions: What We Know So Far. Noncoding RNA. 2019;5:54.

15. Fan YR, Yang JY, Mathioni SM, Yu JS, Shen JQ, Yang XF, Wang L, Zhang QH, Cai ZX, Xu CG, et al. PMS1T, producing phased small-interfering RNAs, regulates photoperiod-sensitive male sterility in rice. P Natl Acad Sci USA. 2016;113:15144-15149. 
16. Ding JH, Lu Q, Ouyang YD, Mao HL, Zhang PB, Yao JL, Xu CG, Li XH, Xiao JH, Zhang QF. A long noncoding RNA regulates photoperiod-sensitive male sterility, an essential component of hybrid rice. P Natl Acad Sci USA. 2012;109:2654-2659.

17. Fang J, Zhang FT, Wang HR, Wang W, Zhao F, Li ZJ, Sun CH, Chen FM, Xu F, Chang SQ, et al. Ef-cd locus shortens rice maturity duration without yield penalty. P Natl Acad Sci USA. 2019;116:18717-18722.

18. Henriques R, Wang $\mathrm{H}$, Liu J, Boix M, Huang LF, Chua NH. The antiphasic regulatory module comprising CDF5 and its antisense RNA FLORE links the circadian clock to photoperiodic flowering. New Phytol. 2017;216:854-867.

19. Severing E, Faino L, Jamge S, Busscher M, Kuijer-Zhang Y, Bellinazzo F, Busscher-Lange J, Fernandez V, Angenent GC, Immink RGH, Pajoro A. Arabidopsis thaliana ambient temperature responsive IncRNAs. Bmc Plant Biol. 2018;18:145.

20. Zhao XY, Li JR, Lian B, Gu HQ, Li Y, Qi YJ. Global identification of Arabidopsis IncRNAs reveals the regulation of MAF4 by a natural antisense RNA. Nat Commun. 2018;9.

21. Shi Y, Zhang X, Xu ZY, Li L, Zhang C, Schlappi M, Xu ZQ. Influence of EARLI1-like genes on flowering time and lignin synthesis of Arabidopsis thaliana. Plant Biology. 2011;13:731-739.

22. Xu B, Sathitsuksanoh N, Tang YH, Udvardi MK, Zhang JY, Shen ZX, Balota M, Harich K, Zhang PYH, Zhao BY. Overexpression of AtLOV1 in Switchgrass Alters Plant Architecture, Lignin Content, and Flowering Time. Plos One. 2012;7: e47399.

23. Cho LH, Yoon J, An G. The control of flowering time by environmental factors. Plant Journal. 2017;90:708-719.

24. Csorba T, Questa JI, Sun QW, Dean C. Antisense COOLAIR mediates the coordinated switching of chromatin states at FLC during vernalization. P Natl Acad Sci USA. 2014;111:16160-16165.

25. Liu FQ, Marquardt S, Lister C, Swiezewski S, Dean C. Targeted 3 ' Processing of Antisense Transcripts Triggers Arabidopsis FLC Chromatin Silencing. Science. 2010;327:94-97.

26. Swiezewski S, Liu FQ, Magusin A, Dean C. Cold-induced silencing by long antisense transcripts of an Arabidopsis Polycomb target. Nature. 2009;462:799-U122.

27. Shin JH, Chekanova JA. Arabidopsis RRP6L1 and RRP6L2 Function in FLOWERING LOCUS C Silencing via Regulation of Antisense RNA Synthesis. PLoS genetics. 2014;10:e1004612.

28. Poole RL. The TAIR database. Methods Mol Biol. 2007;406:179-212.

29. Gallart AP, Pulido AH, de Lagran IAM, Sanseverino W, Cigliano RA. GREENC: a Wiki-based database of plant IncRNAs. Nucleic Acids Res. 2016;44:D1161-D1166.

30. Jia JB, Long YP, Zhang H, Li ZW, Liu ZJ, Zhao Y, Lu DD, Jin XH, Deng X, Xia R, et al. Post-transcriptional splicing of nascent RNA contributes to widespread intron retention in plants. Nat Plants. 2020;6:780788. 
31. Kindgren $\mathrm{P}$, Ivanov $\mathrm{M}$, Marquardt $\mathrm{S}$. Native elongation transcript sequencing reveals temperature dependent dynamics of nascent RNAPII transcription in Arabidopsis. Nucleic Acids Res. 2020;48:23322347.

32. Nielsen $M$, Ard R, Leng $X$, Ivanov $M$, Kindgren $P$, Pelechano $V$, Marquardt S. Transcription-driven chromatin repression of Intragenic transcription start sites. PLoS genetics. 2019;15:e1007969.

33. Thomas QA, Ard R, Liu JH, Li BN, Wang JW, Pelechano V, Marquardt S. Transcript isoform sequencing reveals widespread promoter-proximal transcriptional termination in Arabidopsis. Nat Commun. 2020;11:2589.

34. Kang YJ, Yang DC, Kong L, Hou M, Meng YQ, Wei LP, Gao G. CPC2: a fast and accurate coding potential calculator based on sequence intrinsic features. Nucleic Acids Res. 2017;45:W12-W16.

35. Guo JC, Fang SS, Wu Y, Zhang JH, Chen Y, Liu J, Wu B, Wu JR, Li EM, Xu LY, et al. CNIT: a fast and accurate web tool for identifying protein-coding and long non-coding transcripts based on intrinsic sequence composition. Nucleic Acids Res. 2019;47:W516-W522.

36. Seo JS, Sun HX, Park BS, Huang CH, Yeh SD, Jung C, Chua NH. ELF18-INDUCED LONG-NONCODING RNA Associates with Mediator to Enhance Expression of Innate Immune Response Genes in Arabidopsis. Plant Cell. 2017;29:1024-1038.

37. Kindgren $\mathrm{P}, \mathrm{Ard} \mathrm{R}$, Ivanov $\mathrm{M}$, Marquardt $\mathrm{S}$. Transcriptional read-through of the long non-coding RNA SVALKA governs plant cold acclimation. Nat Commun. 2018;9:4561.

38. Pedersen AG, Nielsen $\mathrm{H}$. Neural network prediction of translation initiation sites in eukaryotes: Perspectives for EST and genome analysis. Fifth International Conference on Intelligent Systems for Molecular Biology, Proceedings. 1997:226-233.

39. Palos K, Dittrich AN, Yu LA, Brock JR, Wu L, Sokolowska E, Skirycz A, Hsu PD, Lyons E, Beilstein MA, Nelson ADL. Identification and Functional Annotation of Long Intergenic Non-coding RNAs in the Brassicaceae. bioRxiv. 2021; doi:10.1101/2021.09.17.460835.

40. Hsu PY, Calviello L, Wu HYL, Li FW, Rothfels CJ, Ohler U, Benfey PN. Super-resolution ribosome profiling reveals unannotated translation events in Arabidopsis. P Natl Acad Sci USA. 2016;113:E7126-E7135.

41. Nelson ADL, Forsythe ES, Devisetty UK, Clausen DS, Haug-Batzell AK, Meldrum AMR, Frank MR, Lyons E, Beilstein MA. A Genomic Analysis of Factors Driving lincRNA Diversification: Lessons from Plants. G3-Genes Genom Genet. 2016;6:2881-2891.

42. Ross CJ, Rom A, Spinrad A, Gelbard-Solodkin D, Degani N, Ulitsky I. Uncovering deeply conserved motif combinations in rapidly evolving noncoding sequences. Genome biology. 2021;22.

43. Beilstein MA, Nagalingum NS, Clements MD, Manchester SR, Mathews S. Dated molecular phylogenies indicate a Miocene origin for Arabidopsis thaliana. P Natl Acad Sci USA. 2010;107:18724-18728. 
44. Jin Y, Marquardt S. Dual sgRNA-based Targeted Deletion of Large Genomic Regions and Isolation of Heritable Cas9-free Mutants in Arabidopsis. Bio-Protocol. 2020;10:e3796.

45. Fedak H, Palusinska M, Krzyczmonik K, Brzezniak L, Yatusevich R, Pietras Z, Kaczanowski S, Swiezewski S. Control of seed dormancy in Arabidopsis by a cis-acting noncoding antisense transcript. P Natl Acad Sci USA. 2016;113:E7846-E7855.

46. Schwab R, Ossowski S, Riester M, Warthmann N, Weigel D. Highly specific gene silencing by artificial microRNAs in Arabidopsis. Plant Cell. 2006;18:1121-1133.

47. Zhou YY, Zhou B, Pache L, Chang M, Khodabakhshi AH, Tanaseichuk O, Benner C, Chanda SK. Metascape provides a biologist-oriented resource for the analysis of systems-level datasets. Nat Commun. 2019;10:1523.

48. Xu H, Handoko L, Wei XL, Ye CP, Sheng JP, Wei CL, Lin F, Sung WK. A signal-noise model for significance analysis of ChIP-seq with negative control. Bioinformatics. 2010;26:1199-1204.

49. Chen W, Salari H, Taylor MC, Jost R, Berkowitz O, Barrow R, Qiu D, Branco R, Masle J. NMT1 and NMT3 N-Methyltransferase Activity Is Critical to Lipid Homeostasis, Morphogenesis, and Reproduction. Plant physiology. 2018;177:1605-1628.

50. Lashbrook CC, Cai S. Cell wall remodeling in Arabidopsis stamen abscission zones: Temporal aspects of control inferred from transcriptional profiling. Plant signaling \& behavior. 2008;3:733-736.

51. Sun LX, van Nocker S. Analysis of promoter activity of members of the PECTATE LYASE-LIKE (PLL) gene family in cell separation in Arabidopsis. Bmc Plant Biol. 2010;10:152.

52. Zhang $X$, Chen Y, Wang ZY, Chen Z, Gu H, Qu LJ. Constitutive expression of CIR1 (RVE2) affects several circadian-regulated processes and seed germination in Arabidopsis. The Plant journal : for cell and molecular biology. 2007;51:512-525.

53. Cai XN, Davis EJ, Ballif J, Liang MX, Bushman E, Haroldsen V, Torabinejad J, Wu YJ. Mutant identification and characterization of the laccase gene family in Arabidopsis. J Exp Bot. 2006;57:2563-2569.

54. Heo JB, Sung S. Vernalization-Mediated Epigenetic Silencing by a Long Intronic Noncoding RNA. Science. 2011;331:76-79.

55. Kim DH, Sung S. Vernalization-Triggered Intragenic Chromatin Loop Formation by Long Noncoding RNAs. Dev Cell. 2017;40:302-312.

56. Capovilla G, Delhomme N, Collani S, Shutava I, Bezrukov I, Symeonidi E, Amorim MD, Laubinger S, Schmid M. PORCUPINE regulates development in response to temperature through alternative splicing. Nat Plants. 2018;4:534-539.

57. Huertas R, Catala R, Jimenez-Gomez JM, Castellano MM, Crevillen P, Pineiro M, Jarillo JA, Salinas J. Arabidopsis SME1 Regulates Plant Development and Response to Abiotic Stress by Determining Spliceosome Activity Specificity. Plant Cell. 2019;31:537-554. 
58. Wang CY, Zhang SC, Yu Y, Luo YC, Liu Q, Ju CL, Zhang YC, Qu LH, Lucas WJ, Wang XJ, Chen YQ. MiR397b regulates both lignin content and seed number in Arabidopsis via modulating a laccase involved in lignin biosynthesis. Plant Biotechnol J. 2014;12:1132-1142.

59. Nakanishi F, Kusumi T, Inoue Y, Fujii T. Dihydrokaempferol Glucoside from Cotyledons Promotes Flowering in Pharbitis-Nil. Plant and Cell Physiology. 1995;36:1303-1309.

60. Hatayama T, Takeno K. The metabolic pathway of salicylic acid rather than of chlorogenic acid is involved in the stress-induced flowering of Pharbitis nil. J Plant Physiol. 2003;160:461-467.

61. Wang Y, Luo XJ, Sun F, Hu JH, Zha XJ, Su W, Yang JS. Overexpressing IncRNA LAIR increases grain yield and regulates neighbouring gene cluster expression in rice. Nat Commun. 2018;9:3516.

62. Ghafouri-Fard S, Dashti S, Taheri M. The HOTTIP (HOXA transcript at the distal tip) IncRNA: Review of oncogenic roles in human. Biomed Pharmacother. 2020;127.

63. Rigo R, Bazin J, Romero-Barrios N, Moison M, Lucero L, Christ A, Benhamed M, Blein T, Huguet S, Charon C, et al. The Arabidopsis IncRNA ASCO modulates the transcriptome through interaction with splicing factors. Embo Rep. 2020;21:e48977.

64. Bardou F, Ariel F, Simpson CG, Romero-Barrios N, Laporte P, Balzergue S, Brown JWS, Crespi M. Long Noncoding RNA Modulates Alternative Splicing Regulators in Arabidopsis. Dev Cell. 2014;30:166-176.

65. Fonouni-Farde C, Ariel F, Crespi M. Plant Long Noncoding RNAs: New Players in the Field of PostTranscriptional Regulations. Non-Coding Rna. 2021;7:12.

66. Groff AF, Sanchez-Gomez DB, Soruco MML, Gerhardinger C, Barutcu AR, Li E, Elcavage L, Plana O, Sanchez LV, Lee JC, et al. In vivo characterization of Linc-p21 reveals functional cis-regulatory DNA elements. Cell Rep. 2016;16:2178-2186.

67. Paralkar VR, Taborda CC, Huang P, Yao Y, Kossenkov AV, Prasad R, Luan J, Davies JOJ, Hughes JR, Hardison RC, et al. Unlinking an IncRNA from its associated cis element. Mol Cell. 2016;62:104-110.

68. Chen J, Brunner AD, Cogan JZ, Nunez JK, Fields AP, Adamson B, Itzhak DN, Li JY, Mann M, Leonetti MD, Weissman JS. Pervasive functional translation of noncanonical human open reading frames. Science. 2020;367:1140-1146.

69. Lewandowski JP, Dumbovic G, Watson AR, Hwang T, Jacobs-Palmer E, Chang N, Much C, Turner KM, Kirby C, Rubinstein ND, et al. The Tug1 IncRNA locus is essential for male fertility. Genome biology. 2020;21:237.

70. Chu C, Quinn J, Chang HY. Chromatin isolation by RNA purification (ChIRP). Journal of visualized experiments : JoVE. 2012.

71. Raj A, van den Bogaard P, Rifkin SA, van Oudenaarden A, Tyagi S. Imaging individual mRNA molecules using multiple singly labeled probes. Nat Methods. 2008;5:877-879. 
72. Nelson ADL, Haug-Baltzell AK, Davey S, Gregory BD, Lyons E. EPIC-CoGe: managing and analyzing genomic data. Bioinformatics. 2018;34:2651-2653.

73. Lyons E, Freeling M. How to usefully compare homologous plant genes and chromosomes as DNA sequences. Plant Journal. 2008;53:661-673.

74. Geneious Prime. https://www.geneious.com. Accessed 2021.

75. Schneider CA, Rasband WS, Eliceiri KW. NIH Image to ImageJ: 25 years of image analysis. Nat Methods. 2012;9:671-675.

76. R Core Team. R: A language and environment for statistical computing. R Foundation for statistical computing, Vienna, Austria. https://www.R-project.org/. accessed.

77. GraphPad Prism 9. GraphPad Software, Inc. . http://www.graphpad.com Accessed 1 Jan 2021

78. Jin Y. FLAIL_2021. https://github.com/Yu-Jin-KU. Accessed 12 Aug 2021.

79. Andrews S. FastQC: a quality control tool for high throughput sequence data. http://www.bioinformatics.babraham.ac.uk/projects/fastqc. Accessed 18 Jan 2019.

80. Bolger AM, Lohse M, Usadel B. Trimmomatic: a flexible trimmer for Illumina sequence data. Bioinformatics. 2014;30:2114-2120.

81. Dobin A, Davis CA, Schlesinger F, Drenkow J, Zaleski C, Jha S, Batut P, Chaisson M, Gingeras TR. STAR: ultrafast universal RNA-seq aligner. Bioinformatics. 2013;29:15-21.

82. Li H, Handsaker B, Wysoker A, Fennell T, Ruan J, Homer N, Marth G, Abecasis G, Durbin R, Proc GPD. The Sequence Alignment/Map format and SAMtools. Bioinformatics. 2009;25:2078-2079.

83. Anders S, Huber W. Differential expression analysis for sequence count data. Genome biology. 2010;11:R106.

84. Krueger F. Trim Galore. https://github.com/FelixKrueger/TrimGalore. Accessed 23 Juy 2021.

85. Yu GC, Wang LG, He QY. ChIPseeker: an R/Bioconductor package for ChIP peak annotation, comparison and visualization. Bioinformatics. 2015;31:2382-2383.

86. Carlson MM, B.P. TxDb.Athaliana.BioMart.plantsmart28: Annotation package for TxDb object(s). https://bioconductor.org/packages/release/data/annotation/html/TxDb.Athaliana.BioMart.plantsm art28.html. Accessed 7 Oct 2015.

87. Robinson JT, Thorvaldsdottir H, Winckler W, Guttman M, Lander ES, Getz G, Mesirov JP. Integrative genomics viewer. Nature biotechnology. 2011;29:24-26.

88. Jin Ivanov $\mathrm{Y}$, M, Marquardt https://www.ncbi.nlm.nih.gov/geo/query/acc.cgi?acc=GSE186215. Accessed 20 Oct 2021. 
bioRxiv preprint doi: https://doi.org/10.1101/2021.11.15.468639; this version posted November 16, 2021. The copyright holder for this Fig. preprint (which was not certified by peer review) is the author/funder, who has granted bioRxiv a license to display the preprint in perpetuity. It is made available under aCC-BY-NC-ND 4.0 International license.

A

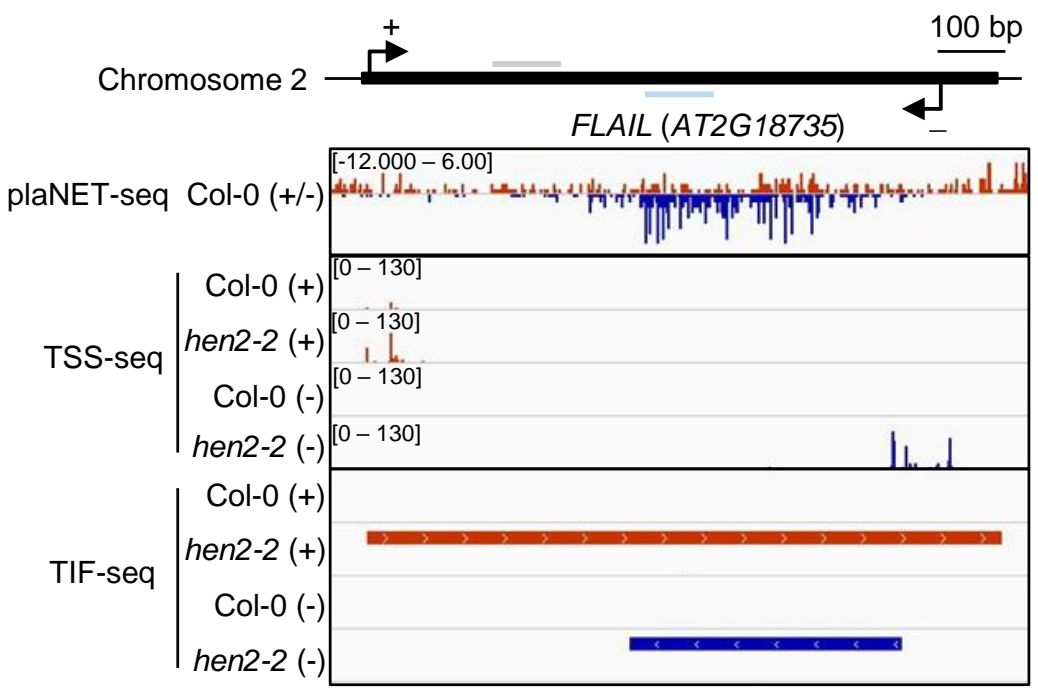

\section{C}

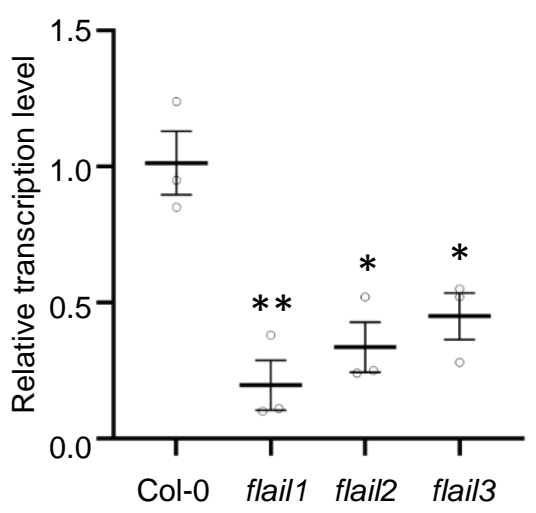

E

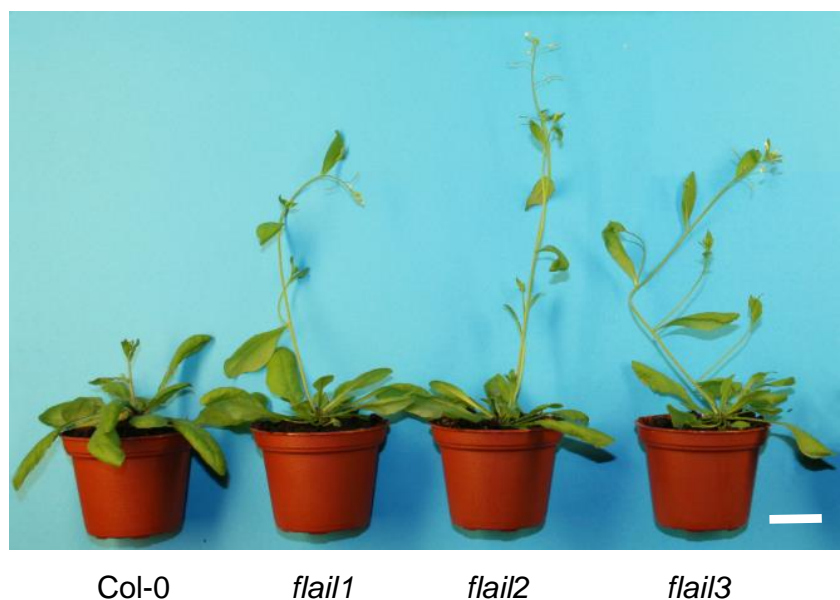

B

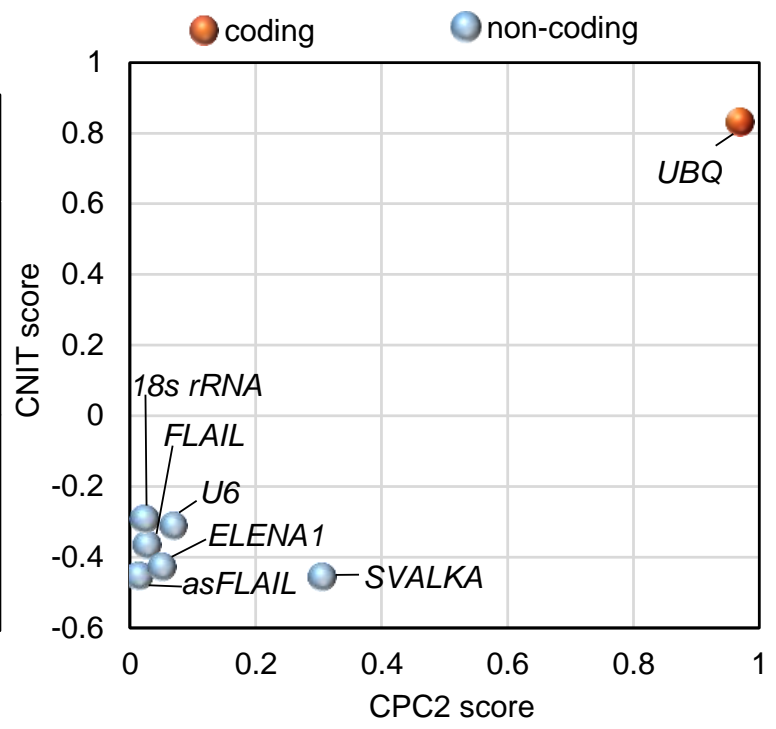

D

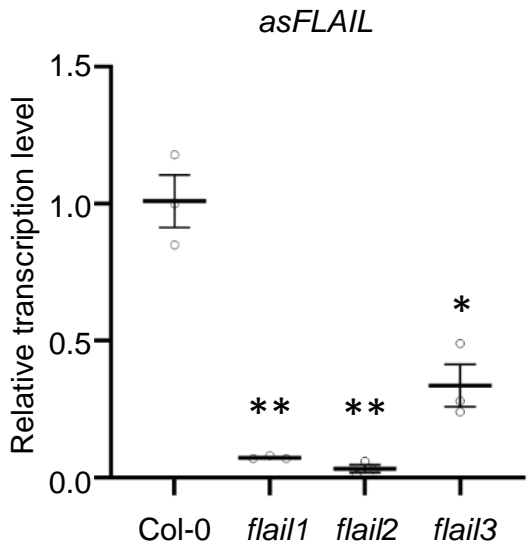

$\mathbf{F}$

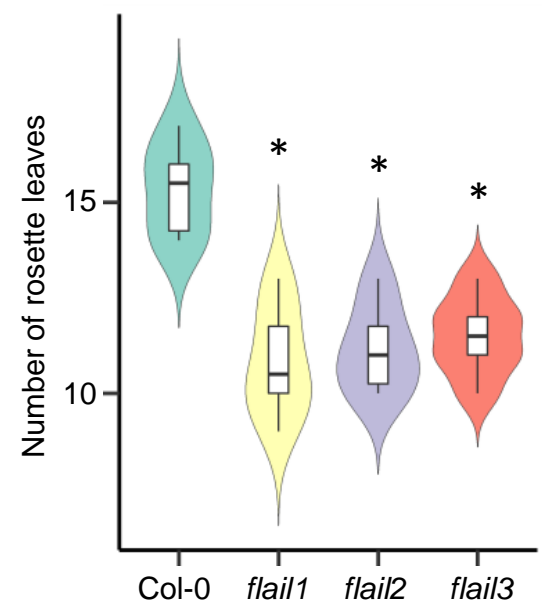




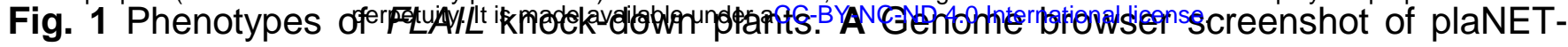

seq, TSS-seq and TIF-seq at the FLAIL genomic region in Col-0 and hen2-2 mutant. Sense (+) and antisense (-) strands were shown in red and dark blue, respectively. Grey bar and light blue bar indicated primer locations of RT-qPCR for sense FLAIL (FLAIL) and antisense FLAIL (asFLAIL), respectively. B Coding potential of the transcript in the genomic region of $F L A / L$ and asFLAIL and reference transcripts including non-coding RNAs (18sRNA, U6, ELENA1, SVALKA) and coding gene UBQ according to the CNIT and CPC2 algorithm. C-D Detection of FLAIL and asFLAIL gene expression in Col-0 and flail mutants by RT-qPCR. Transcript levels were normalized to $U B Q$ expression levels. Y-axis showed relative values compared to the expression level of Col-0. Bars represented average \pm s.e.m $(n=3$ independent 14-d seedling pools). ${ }^{*}, p$ value $<0.05$ and ${ }^{* *}, p$ value $<0.01$ by Student's t-test compared to Col-0. $\mathbf{E}$ Morphological phenotypes of 4-week-old plants of Col-0, flail mutants at $20{ }^{\circ} \mathrm{C}$ under a $16-\mathrm{h}$ light/8-h dark growth condition. Scale bar: $2 \mathrm{~cm}$. F Violin graph showed number of rosette leaves after appearance of the first flower bud in Col-0. Data represented the mean of six independent experiments. Boxes spanned the first to third quartile, bold black lines indicated median value for each group and whiskers represented the minimum and maximum values. *, $p$ value $<0.05$ was indicated by Student's t-test compared to Col-0. 
A
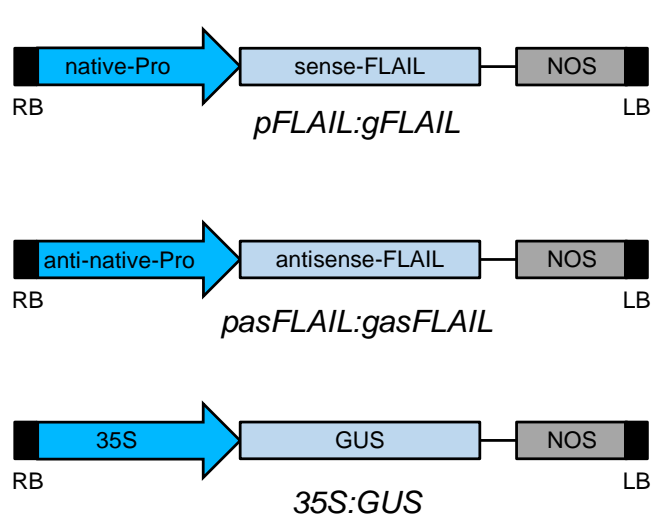

B

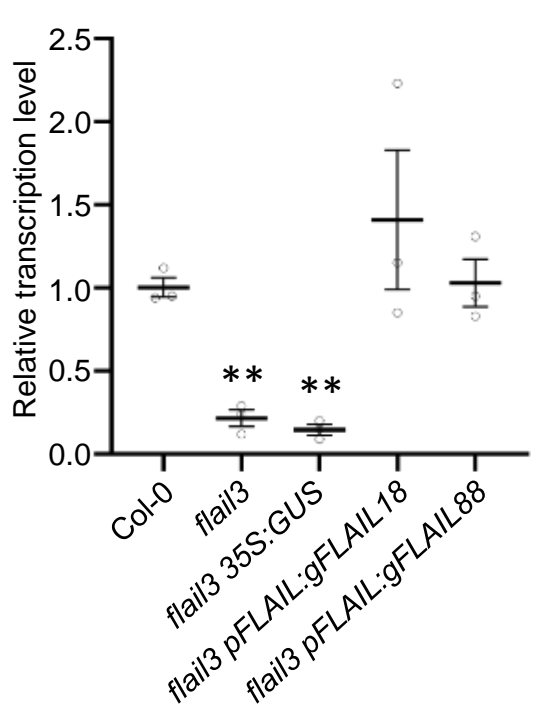

FLAIL
C

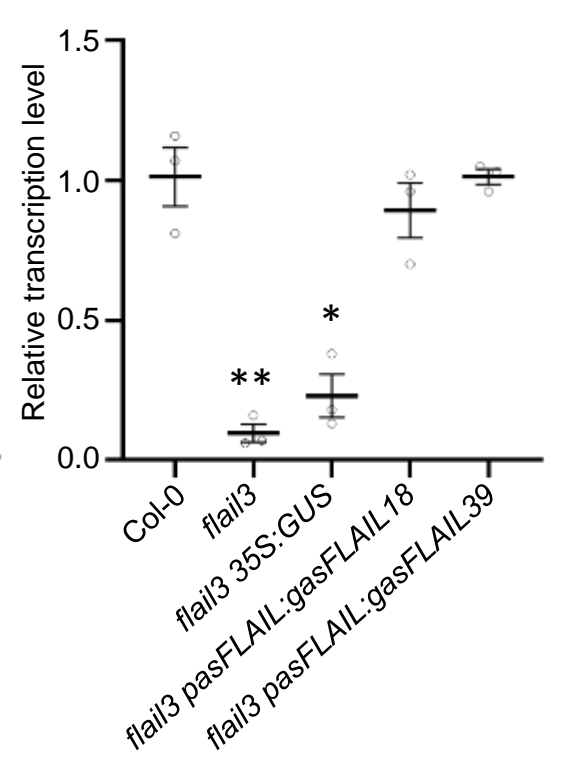

E

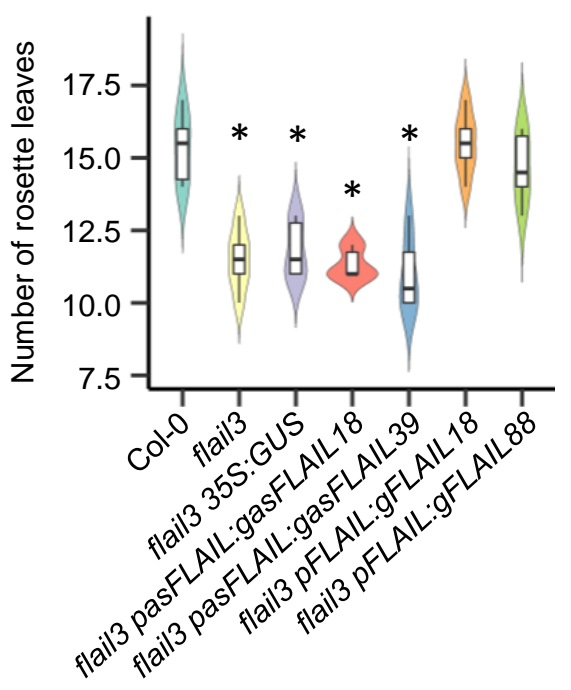

D

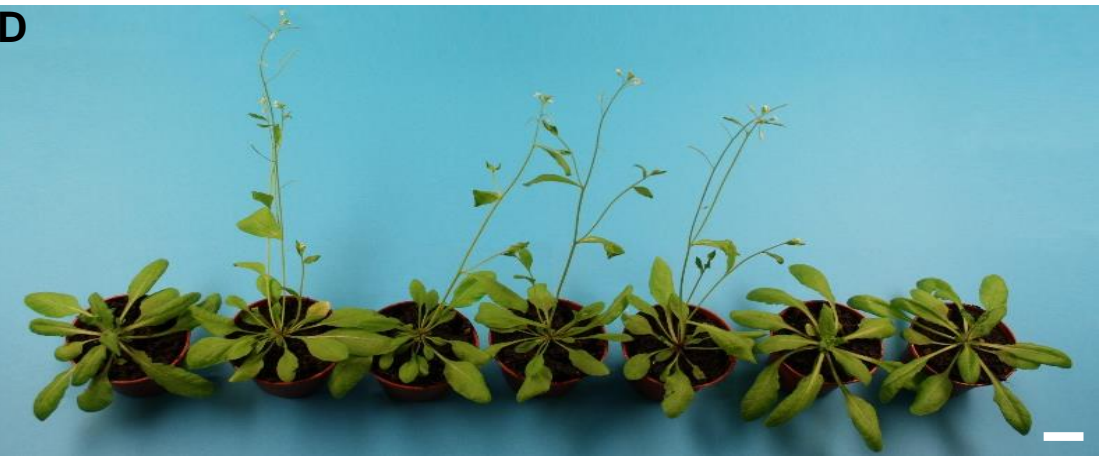

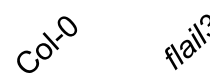<smiles>CC(C)C</smiles>

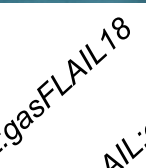<smiles>N#CCOCC(=O)O</smiles>

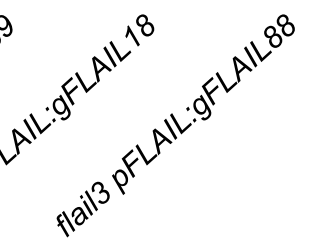




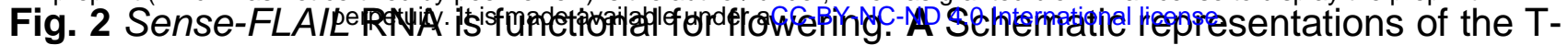

DNA constructs containing the native promoter of sense-FLAIL (native-Pro) fused to the sense-FLAIL DNA region with NOS as a terminator or the native promoter of anti-sense FLAIL (anti-native-Pro) fused to the anti-sense FLAIL DNA region or a negative control with the $35 S$ promoter (35S) fused to GUS reporter were transformed into flail3 Arabidopsis plants. B-C Detection of FLAIL and asFLAIL genes expression in Col-0, flail3 mutant and complemented lines expressing pFLAIL:gFLAIL and pasFLAIL:gasFLAIL by RT-qPCR. Transcript levels were normalized to $U B Q$ expression levels. Y-axis showed relative values compared to the expression level of Col-0. Error bars represented s.e.m (n $=3$ independent $14-d$ seedling pools). ${ }^{*}, p$ value $<0.05$ and ${ }^{* *}, p$ value $<0.01$ by Student's t-test compared to Col-0. D Representative morphological phenotypes of 4-week-old plants of Col-0, flail3 mutant, transgenic lines at $20^{\circ} \mathrm{C}$ under a 16-h light/8-h dark growth condition. Scale bar: $2 \mathrm{~cm}$. E Violin graph showed number of rosette leaves after appearance of the first flower bud in Col-0. Data represented the mean of six independent experiments. Boxes spanned the first to third quartile, bold black lines indicated median value for each group and whiskers represented the minimum and maximum values. ${ }^{*}, p$ value $<0.05$ was indicated by Student's t-test compared to Col- 0 . 
bioRxiv preprint doi: https://doi.org/10.1101/2021.11.15.468639; this version posted November 16, 2021. The copyright holder for this Fig reprint (which was not certified by peer review) is the author/funder, who has granted bioRxiv a license to display the preprint in

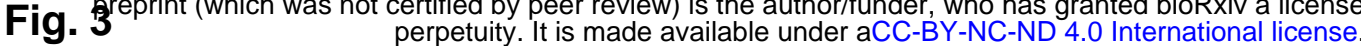

A

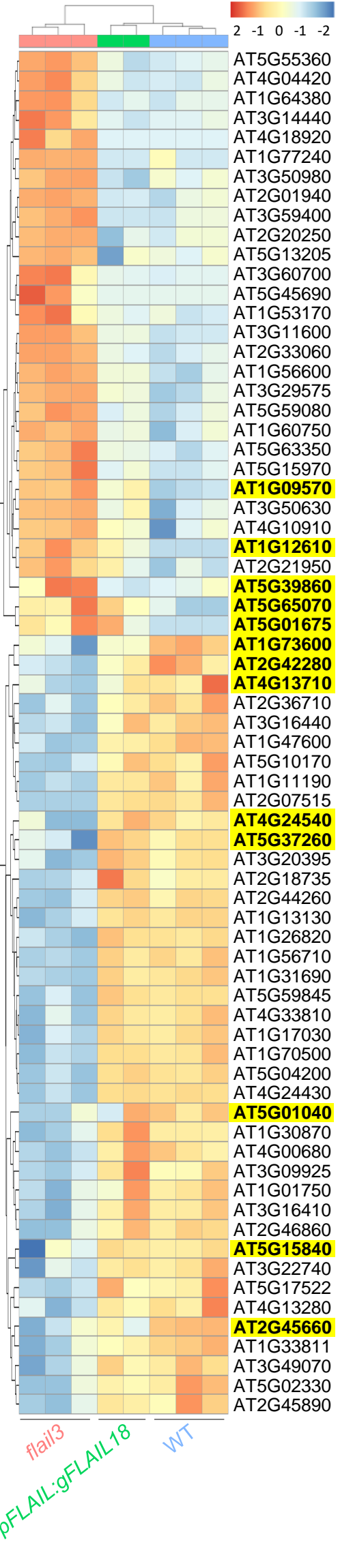

B

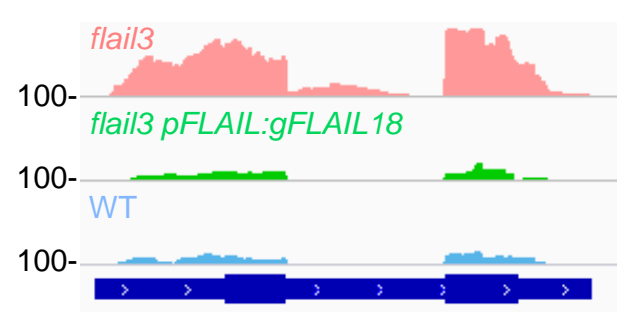

AT5G39860
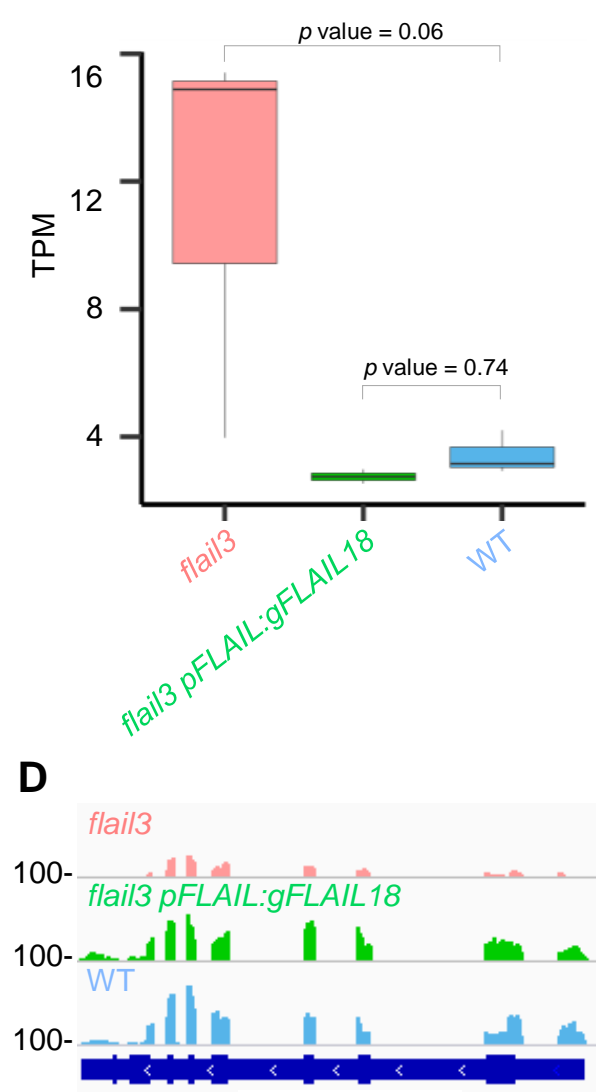

AT4G24540

$p$ value $=0.011$

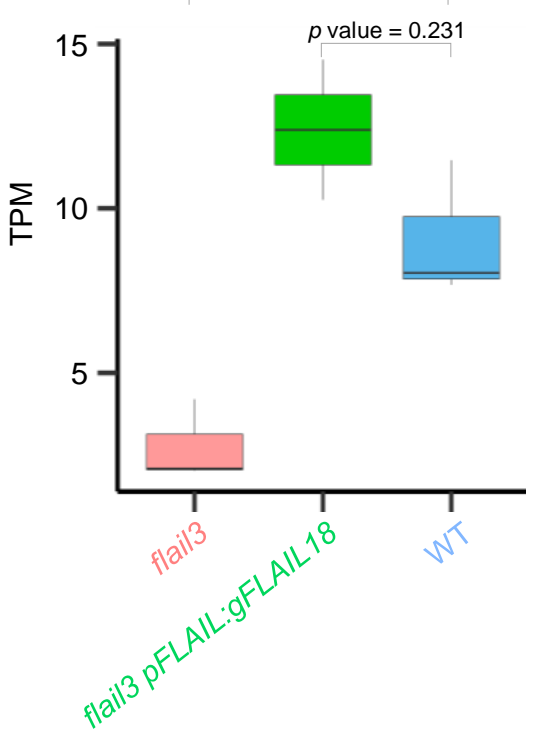

C $500 \mathrm{bp}$

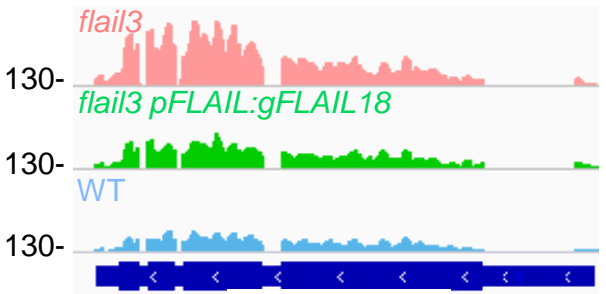

AT1G09570

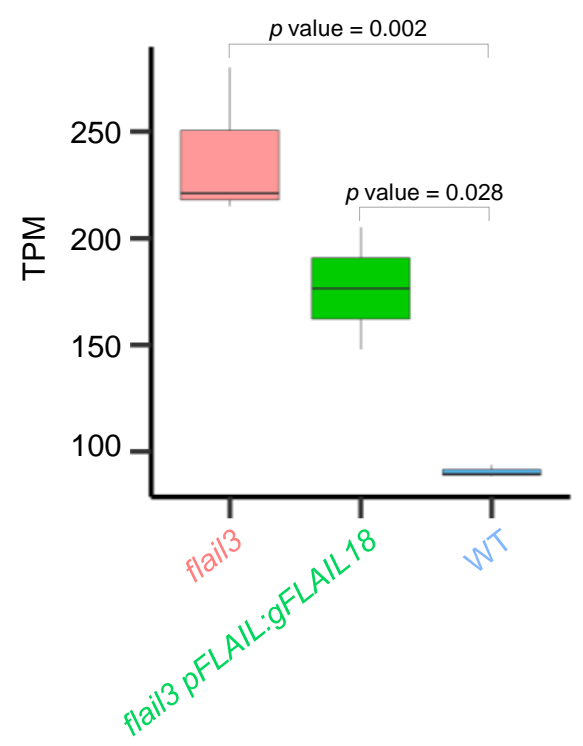

E

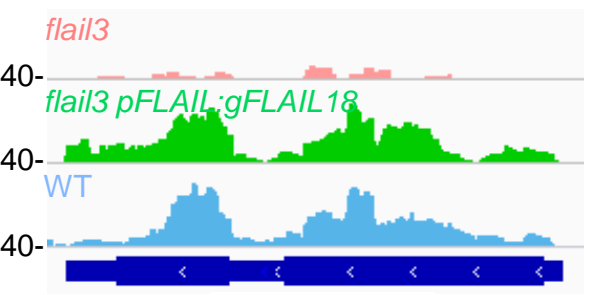

AT5G15840

$p$ value $=0.004$

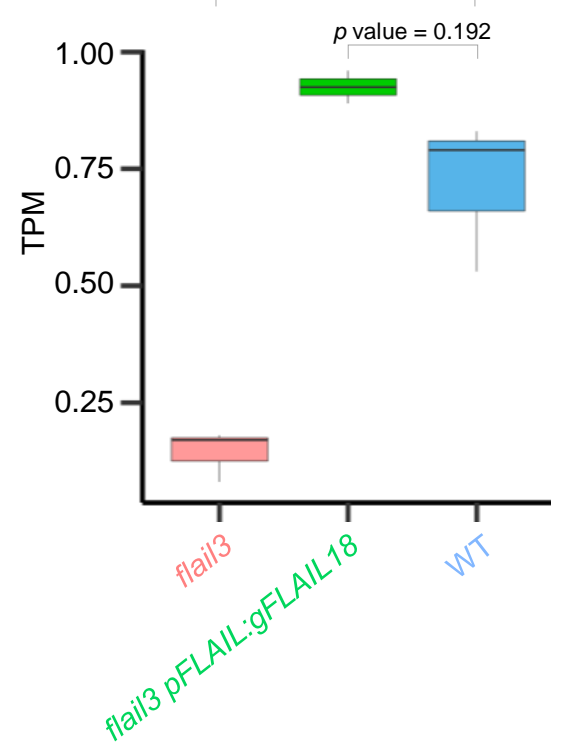




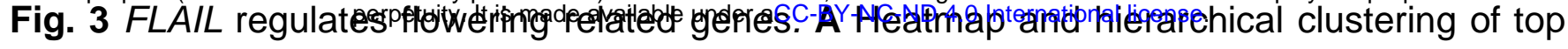

70 differentially expressed genes (DEGs) in flail3 versus Col-0 that were best rescued in the flail3 pFLAIL:gFLAIL18 complementation line. DEGs analyzed by DESeq2 with |log2 fold change $\mid>1$ and adjusted $p$ value $<0.05$ were considered significant differential expression. Three biological replicates for flail3, wild type, and two for flail3 pFLAIL:gFLAIL18. Flowering genes were highlighted in yellow bold. Samples clustered together on the basis of corresponding similar expression profiles. The color scale reflected the log 2-fold change in gene expression, ranging from down-regulated (blue) to up-regulated (red). B-E Genome browser screenshots illustrating the expression of dysregulated flowering genes in flail3 were rescued in complementation line (top panel). Normalized read counts (TPM from RNA-seq) for differentially expressed flowering genes in WT, flail3, and flail3 pFLAIL:gFLAIL18 plants (bottom panel). Boxes spanned the first to third quartile, bold black lines indicated median value for each group and whiskers represented the minimum and maximum values. All $p$ values were denoted by Students' t-test. Bar $=500 \mathrm{bp}$. 
bioRxiv preprint doi: https://doi.org/10.1101/2021.11.15.468639; this version posted November 16, 2021. The copyright holder for this Fig. 4 reprint (which was not certified by peer review) is the author/funder, who has granted bioRxiv a license to display the preprint in perpetuity. It is made available under aCC-BY-NC-ND 4.0 International license.

A

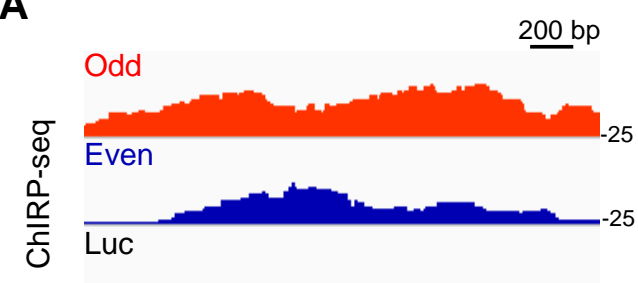

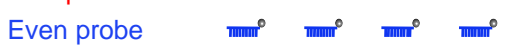

FLAIL (sense RNA)

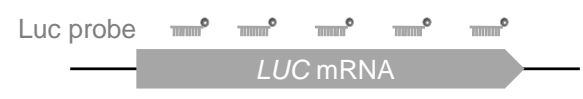

D

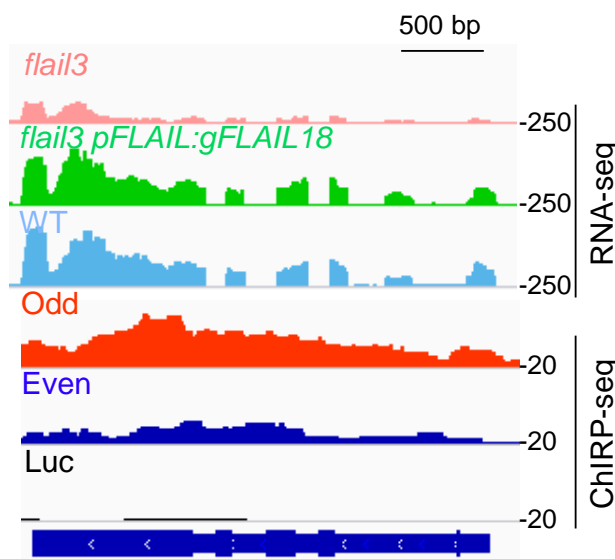

AT5G37260 (CIR1)

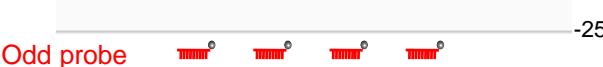

B

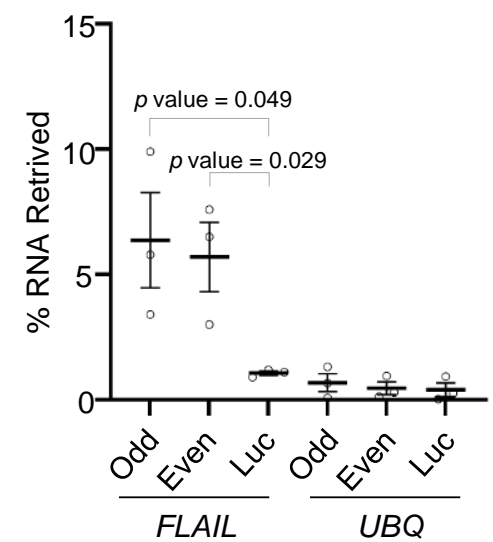

E

\section{AT5G37260 (CIR1)}

$p$ value $=0.011$

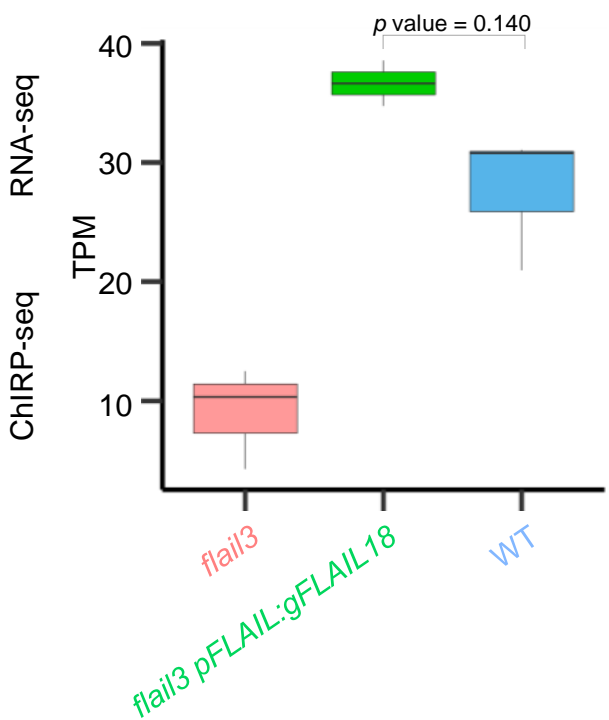

G

$500 \mathrm{bp}$

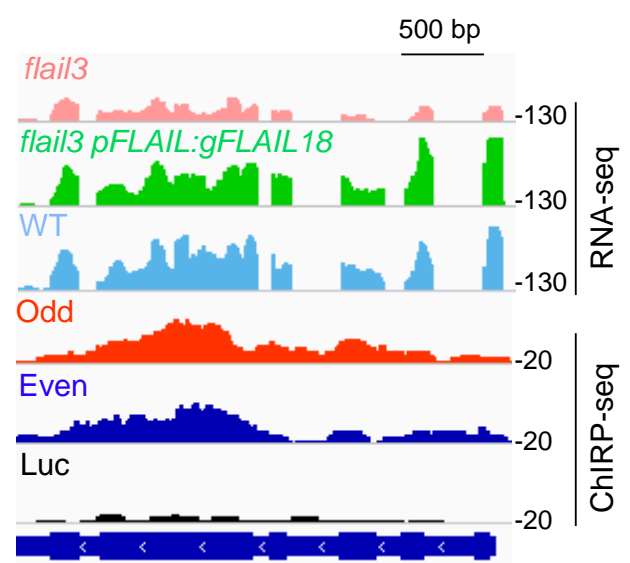

AT5G01040 (LAC8)
H

AT5G01040 (LAC8)

$p$ value $=0.006$

$p$ value $=0.067$

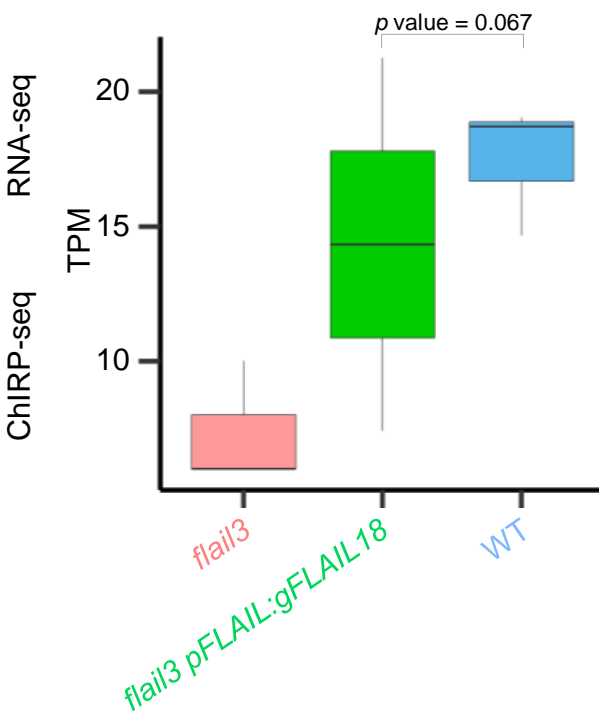

C

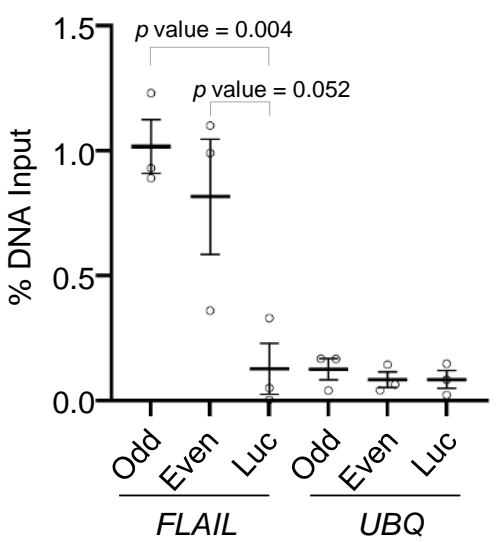

F

DEGs in flail3 ChIRP-seq

flowering genes

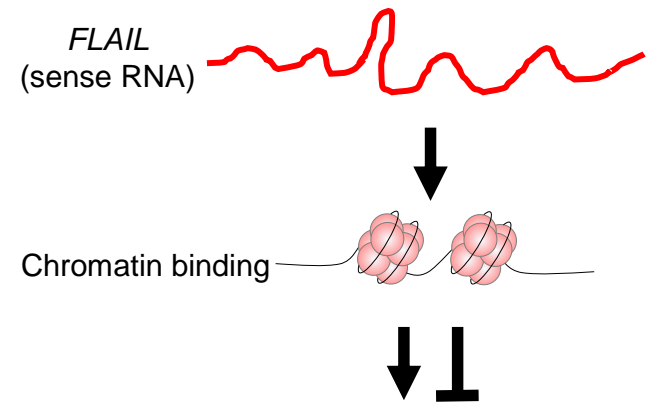

Gene expression
Flowering genes

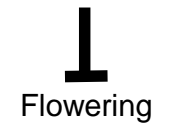




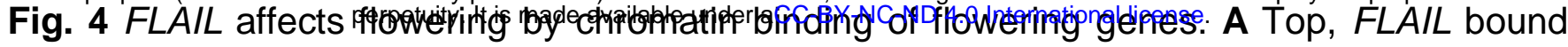
the locus itself by ChIRP-seq from two independent Odd and Even probed chromatins. Luc probe was used as a control. Bottom, schematic representation of the antisense oligonucleotide probes that were biotinylated at the $3^{\prime}$-end with Odd (in red) and Even (in dark blue) against FLAIL sense RNA and Luc probe (in grey) against LUC mRNA. B ChIRP-qPCR using probe pools FLAIL-asDNA (Odd and Even) retrieved $~ 5 \%-10 \%$ of FLAIL endogenous RNA and $<1 \%$ levels of $U B Q$. Luc-asDNA probes retrieved much lower levels of both RNAs as a control. C FLAIL DNA signal was identified in both Odd and Even probes. UBQ region showed much less binding signal in all probes as a negative control. Graphs in B \& C showed the mean \pm s.e.m. ( $n=3$ independent replicates). D, G Genome browser screenshots illustrating two of FLAIL bound targets CIR1 (D) and LAC8 (G) in RNA-seq (lane 1-3) and ChIRP-seq (lane 4-6). FLAIL binding peaks were called by CCAT3.0 with the cutoff FDR = 0.232. E, H Normalized read counts (TPM from RNA-seq) for differentially expressed (DE) flowering genes in WT, flail3, and flail3 pFLAIL:gFLAIL18 plants (bottom panel). Boxes spanned the first to third quartile, bold black lines indicated median value for each group and whiskers represented the minimum and maximum values. $\mathbf{F}$ Venn diagram of genes targeted by FLAIL (ChIRP-seq) and genes overlapped with DEGs in flail3 and flowering related genes. I A model for the trans-acting FLAIL sense RNA regulated flowering. The FLAIL sense RNA binds chromatin regions of flowering genes to regulate expression levels of flowering related genes and thus affects flowering time. All $p$ values were denoted by Students' $t$-test. 


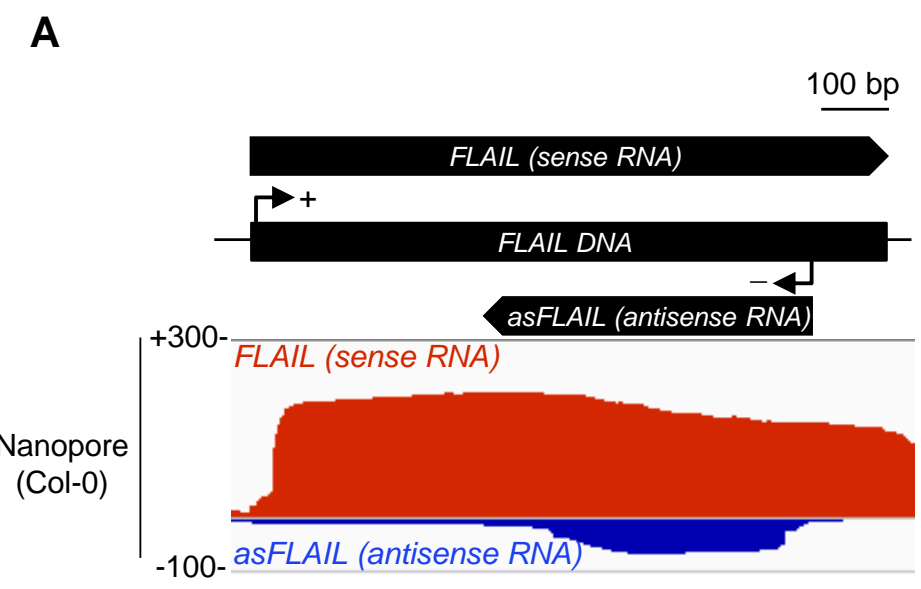

B

AAGTTAATAAACCACACAAAAAAGTTTTTCTCTCTTCGCTCTCTCGTAGTTTGTAAAGATAGAAACTGAAATTTCTTTTGTTTTTGTTAACCGG ATAGTTTTCCGGTGAGTCGCCGTCCCGTTTAATTCGGGCTTTCGTCCGACATCTTCTAGCTACGTTAGTGGAGATGGTTGGCTTTTTGTATGGC ATCGTTGGCTAACTTAGCGACGTTGGCCAGCTTCCGTACGGCAGCGTTGGGCTTACACTAACAGCGTTGGTCATCTTTGTTTTTGTTTTGTTAC TTCAAAGGTTTGATGATGAAGCTTTATGGCATGCAGAATCGTCAGATCTGAAGAGGGTTTCTCTTTACAAGATCTATTCTCCTTCTGTTTTGTT TAGCTTCACTATTGGGTTATGCTTTTGGCTCTGGTAGTTTTTCGGCGATGTGTGCGTTTCCAGCAATTACCCTCGTTTGGATCTACCATATTTA AGACAGTTTCAATTTTATTTTCGCCTAAGATTGGGTTAAAGCTTCTATCCTATGCAAATTTGATATGCCTTTAGTGCTTTTGCAAGAGAGTTCG AAGATTCAATGGTTGGACAAGTTTGAACTACTCAATTTTTTTAATTTCTTTACCCGTTTTGATGAATTATTTGTGATTCTAGCAACCATATGAG ATTAGGAAATTGATGTTTGTTCTATAACATCTAATTGCCATAATCGGTGATAGAAAAAAAGAGTTTCATTGCTCGGTGCTTTCCCGGCGATGGT TCTCCGATTCACCAGACTTGAGTCTCATCCGGTGGAGGTTGTGGAAGATTCACGTGTCCTTAGCTTATTATTATTGGTCTGACACGTCTTAAGC TTATCTCTTTTTTGTGACGTAAAAGTCTGTTGTGGACCTCATATGTAGACTGTCTTGAGCCTTTTATGTTGTAACACTGAGCCTCAGTATATTA AAAATAAATCTTTCTCTG

Fig. S1 Characterization of FLAIL locus. A Genome browser view of FLAIL splicing status in nanopore sequencing of Col-0. Transcription of sense FLAIL RNA and asFLAIL RNA were shown in red and dark blue, respectively and no isoform resulting from alternative splicing was observed. B FLAIL nucleotide sequences in black and two sORFs locations in blue. 


\section{NetStart Prediction}

Name: FLAIL

AAGTTAATAAACCACACAAAAAGTTTTTCTCTCTTCG CTCTCTCGTAGTTTGTAAAGATAGAAACTGAAATTTCT TTTGTTTTTGTTAACCGGATAGTTTTCCGGTGAGTCGC CGTCCCGTTTAATTCGGGCTTTCGTCCGACATCTTCTA GCTACGTTAGTGGAGATGGTTGGCTTTTTGTATGGCAT CGTTGGCTAACTTAGCGACGTTGGCCAGCTTCCGTACG GCAGCGTTGGGCTTACACTAACAGCGTTGGTCATCTTT GTTTTTGTTTTGTTACTTCAAAGGTTTGATGATGAAGC TTTATGGCATGCAGAATCGTCAGATCTGAAGAGGGTTT CTCTTTACAAGATCTATTCTCCTTCTGTTTTGTTTAGC TTCACTATTGGGTTATGCTTTTGGCTCTGGTAGTTTTT CGGCGATGTGTGCGTTTCCAGCAATTACCCTCGTTTGG ATCTACCATATTTAAGACAGTTTCAATTTTATTTTTGC CTAAGATTGGGTTAAAGCTTCTATCCTATGCAAATTTG ATATGCCTTTAGTGCTTTTGCAAGAGAGTTCGAAGATT CAATGGTTGGACAAGTTTGAACTACTCAATTTTTTTAA TTTCTTTACCCGTTTTGATGAATTATTTGTGATTCTAG CAACCATATGAGATTAGGAAATTGATGTTTGTTCTATA ACATCTAATTGCCATAATCGGTGATAGAAAAAAAGAGT TTCATTGCTCGGTGCTTTCCCGGCGATGGTTCTCCGAT TCACCAGACTTGAGTCTCATCCGGTGGAGGTTGTGGAA GATTCACGTGTCCTTAGCTTATTATTATTGGTCTGACA CGTCTTAAGCTTATCTCTTTTTTTGTGACGTAAAAGTCT GTTGTGGACCTCATATGTAGACTGTCTTGAGCCTTTTA TGTTGTAACACTGAGCCTCAGTATATTAAAAATAAATC TTTCTCTG
Name: UBQ10

TCGTGTTTATCAACTCAAAGCACAAATACTTTTTCCTCAACCTAAAAATAAGGCAATTAGCCAAAACAACTTTGGGTGTAAACAACGCTCAATACAC GTGTCATTTTATTATTAGCTATTGCTTCACCGCCTTAGCTTTCTCGTGACCTAGTCGTCCTCGTCTTTTCTTCTTCTTCTTCTATAAAACAATACCC AAAGAGCTCTTCTTCTTCACAATTCAGATTTCAATTTCTCAAAATCTTAAAAACTTTCTCTCAATTCTCTCTACCGTGATCAAGGTAAATTTCTGTG TTCCTTATTCTCTCAAAATCTTCGATTTTGTTTTCGTTCGATCCCAATTTCGTATATGTTCTTTGGTTTAGATTCTGTTAATCTTAGATCGAAGACG ATTTTCTGGGTTTGATCGTTAGATATCATCTTAATTCTCGATTAGGGTTTCATAGATATCATCCGATTTGTTCAAATAATTTGAGTTTTGTCGAATA ATTACTCTTCGATTTGTGATTTCTATCTAGATCTGGTGTTAGTTTCTAGTTTGTGCGATCGAATTTGTCGATTAATCTGAGTTTTTCTGATTAACAG ATGCAGATCTTTGTTAAGACTCTCACCGGAAAGACAATCACCCTCGAGGTGGAAAGCTCCGACACCATCGACAACGTTAAGGCCAAGATCCAGGATA AGGAGGGCATTCCTCCGGATCAGCAGAGGCTTATTTTCGCCGGCAAGCAGCTAGAGGATGGCCGTACGTTGGCTGATTACAATATCCAGAAGGAATC CACCCTCCACTTGGTCCTCAGGCTCCGTGGTGGTATGCAGATTTTTCGTTAAAACCCTAACGGGAAAGACGATTACTCTTGAGGTGGAGAGTTCTGAC ACCATCGACAACGTCAAGGCCAAGATCCAAGACAAAGAGGGTATTCCTCCGGACCAGCAGAGGCTGATCTTCGCCGGAAAGCAGTTGGAGGATGGCA GAACTCTTGCTGACTACAATATCCAGAAGGAGTCCACCCTTCATCTTGTTCTCAGGCTCCGTGGTGGTATGCAGATTTTCGTTAAGACGTTGACTGG GAAAACTATCACTTTGGAGGTGGAGAGTTCTGACACCATTGATAACGTGAAAGCCAAGATCCAAGACAAAGAGGGTATTCCTCCGGACCAGCAGAGA TTGATCTTCGCCGGAAACAACTTGAAGATGGCAGAACTTTGGCCGACTACAACATTCAGAAGGAGTCCACACTCCACTTGGTCTTGCGTCTGCGTG GAGGTATGCAGATCTTCGTGAAGACTCTCACCGGAAAGACCATCACTTTGGAGGTGGAGAGTTCTGACACCATTGATAACGTGAAAGCCAAGATCCA GGACAAAGAGGGTATCCCACCGGACCAGCAGAGATTGATCTTCGCCGGAAAGCAACTTGAAGATGGAAGAACTTTGGCTGACTACAACATTCAGAAG GAGTCCACACTTCACTTGGTCTTGCGTCTGCGTGGAGGTATGCAGATCTTCGTGAAGACTCTCACCGGAAAGACTATCACTTTGGAGGTAGAGAGCT CTGACACCATTGACAACGTGAAGGCCAAGATCCAGGATAAGGAAGGAATCCCTCCGGACCAGCAGAGGTTGATCTTTGCCGGAAAACAATTGGAGGA TGGTCGTACTTTGGCGGATTACAACATCCAGAAGGAGTCGACCCTTCACTTGGTGTTGCGTCTGCGTGGAGGTATGCAGATCTTCGTCAAGACTTTG ACCGGAAAGACCATCACCCTTGAAGTGGAAAGCTCCGACACCATTGACAACGTCAAGGCCAAGATCCAGGACAAGGAAGGTATTCCTCCGGACCAGC AGCGTCTCATCTTCGCTGGAAAGCAGCTTGAGGATGGACGTACTTTGGCCGACTACAACATCCAGAAGGAGTCTACTCTTCACTTGGTCCTGCGTCT TCGTGGTGGTTTCTAAATCTCGTCTCTGTTATGCTTAAGAAGTTCAATGTTTCGTTTCATGTAAAACTTTGGTGGTTTGTGTTTTGGGGCCTTGTAT AATCCCTGATGAATAAGTGTTCTACTATGTTTCCGTTCCTGTTATCTCTTTCTTTCTAATGACAAGTCGAACTTCTTCTTTATCATCGCTTCGTTTT TATTATCTGTGCTTCTTTTGTTTAATACGCCTGCAAAGTGACTCGACTCTGTTTAGTGCAGTTCTGCGAAACTTGTAAATAGTCCAATTGTTGGCCT CTAGTAATAGATGTAGCGAAAGTGTTGAGCTGTTGGGTTCTAAGGATGGCTTGAACATGTTAATCTTTTAGGTTCTGAGTATGATGAACATTCGTTG TTGC
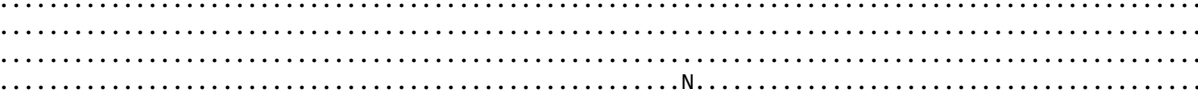

…

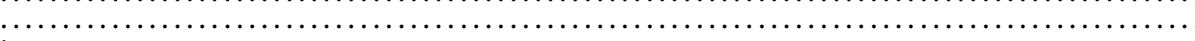

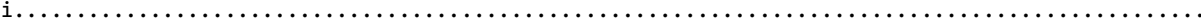
 …

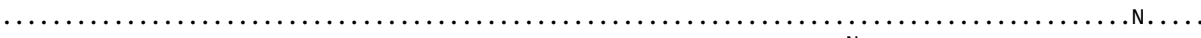

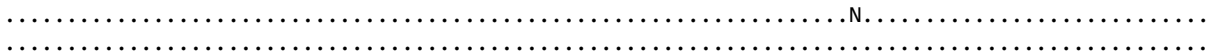

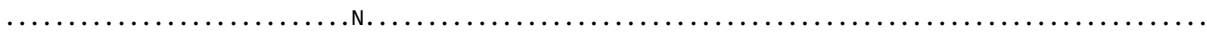

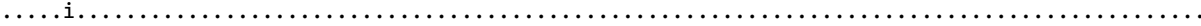

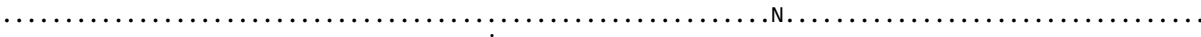

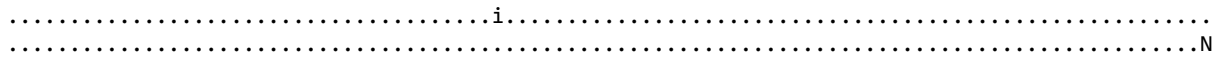

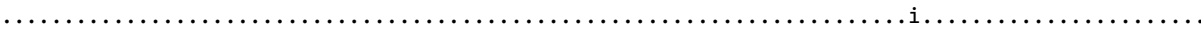

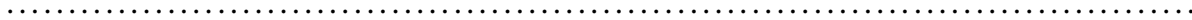

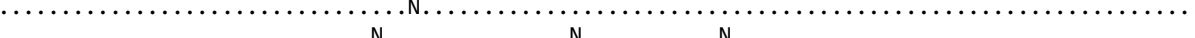

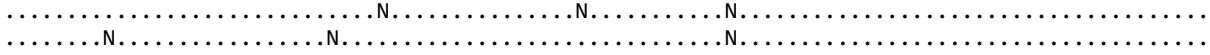
 (1)

Pos Score Pred

$\begin{array}{ccc}\text { Pos } & \text { Score } & \text { Pred } \\ 168 & 0.419 & - \\ 184 & 0.218 & - \\ 295 & 0.106 & - \\ 298 & 0.149 & - \\ 308 & 0.366 & - \\ 313 & 0.287 & - \\ 395 & 0.318 & - \\ 424 & 0.324 & - \\ 522 & 0.290 & - \\ 535 & 0.346 & - \\ 573 & 0.305 & - \\ 626 & 0.262 & - \\ 654 & 0.199 & - \\ 671 & 0.443 & - \\ 748 & 0.208 & - \\ 889 & 0.080 & - \\ 912 & 0.048 & -\end{array}$

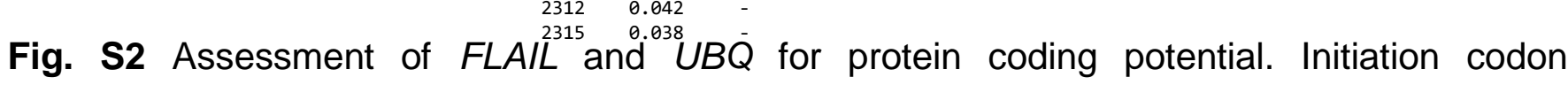
translational analysis using NetStart for FLAIL and UBQ. The predicted initiation codons were depicted with the letter $\mathrm{i}$, other instances of "ATG" by the letter "N" (non-start). The dots (".") were place holders for all the other sequence elements. The scores were always in [0.0, 1.0]; when greater than 0.5 , they represented a probable translation start. 
bioRxiv preprint doi: https://doi.org/10.1101/2021.11.15.468639; this version posted November 16, 2021. The copyright holder for this Fig. 53 (which was not certified by peer review) is the author/funder, who has granted bioRxiv a license to display the preprint in
perpetuity. It is made available under aCC-BY-NC-ND 4.0 International license.

A

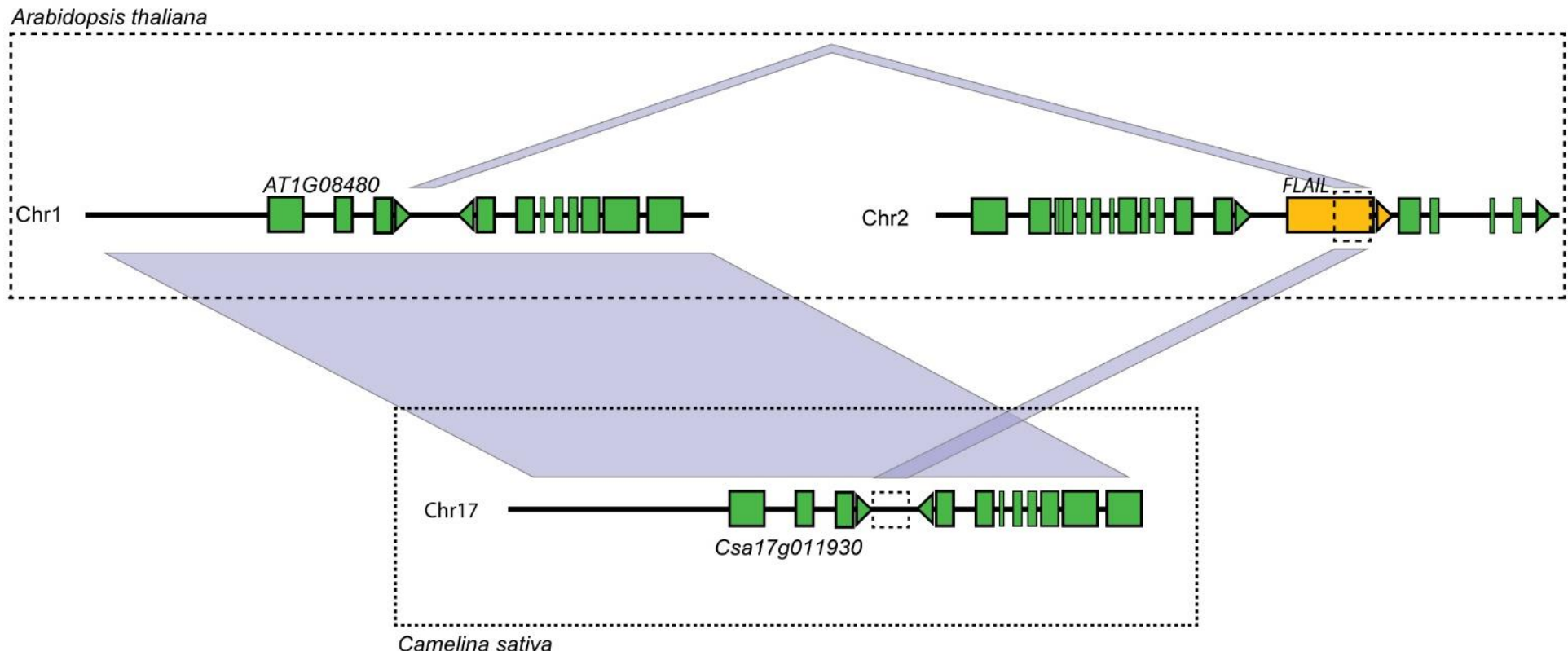

B

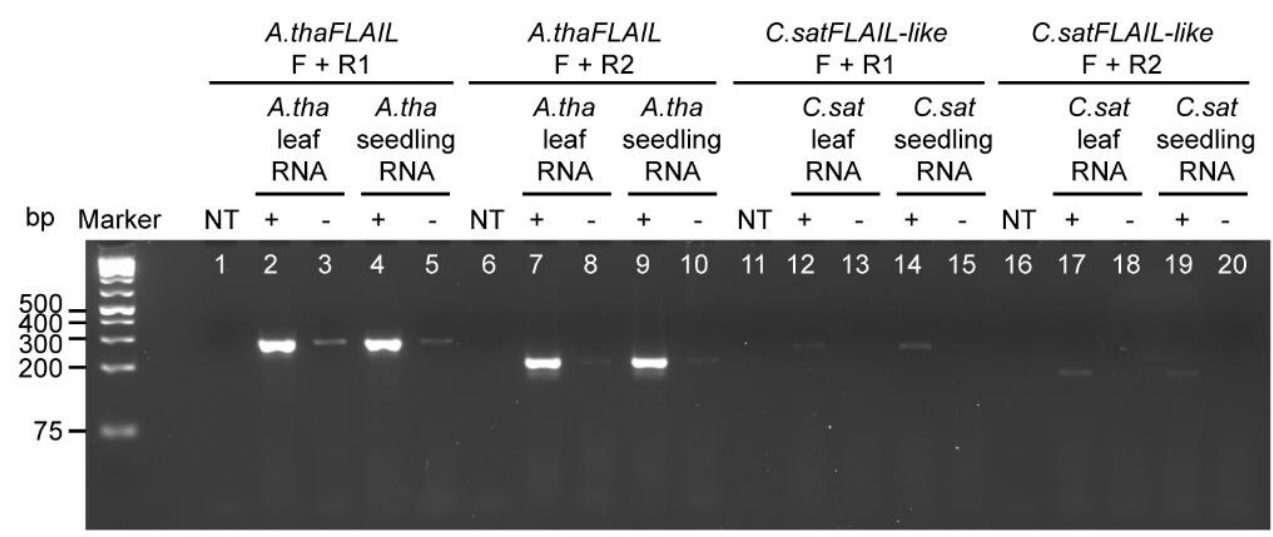

C

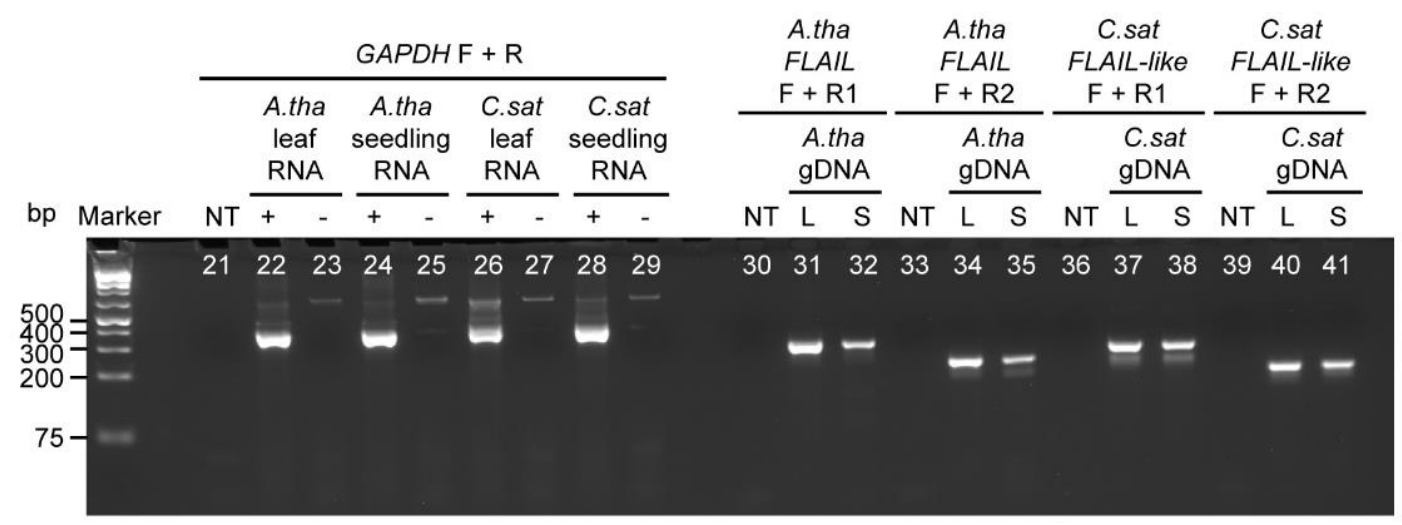




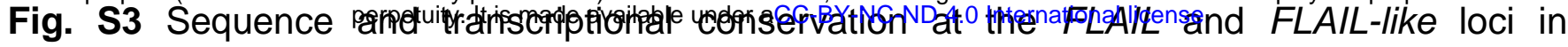

Arabidopsis and Camelina. A Schematic depicting the conservation of the Arabidopsis (A.tha) FLAIL locus in Camelina sativa (C.sat). Green boxes represent exons, with triangles representing direction of transcription. The FLAIL locus is represented by a yellow box. Faded blue lines represent sequence similarity between different loci. Dashed boxes at the FLAIL and Csa17g011930 loci represent regions targeted for RT-PCR. B Amplification of A.thaFLAIL and C.satFLAIL-like, using RNA template (+/- RT, lanes 1-20). NT indicates no template was added to the reaction. C Control amplifications: GAPDH was amplified using RNA template (+/- RT, lanes 21-29), and A.thaFLAIL and C.satFLAIL-like were amplified using genomic DNA template (lanes 30-41). $L$ indicates leaf tissue, $S$ indicates seedling tissue. 
bioRxiv preprint doi: https://doi.org/10.1101/2021.11.15.468639; this version posted November 16, 2021. The copyright holder for this Fig ergprint (which was not certified by peer review) is the author/funder, who has granted bioRxiv a license to display the preprint in perpetuity. It is made available under aCC-BY-NC-ND 4.0 International license.

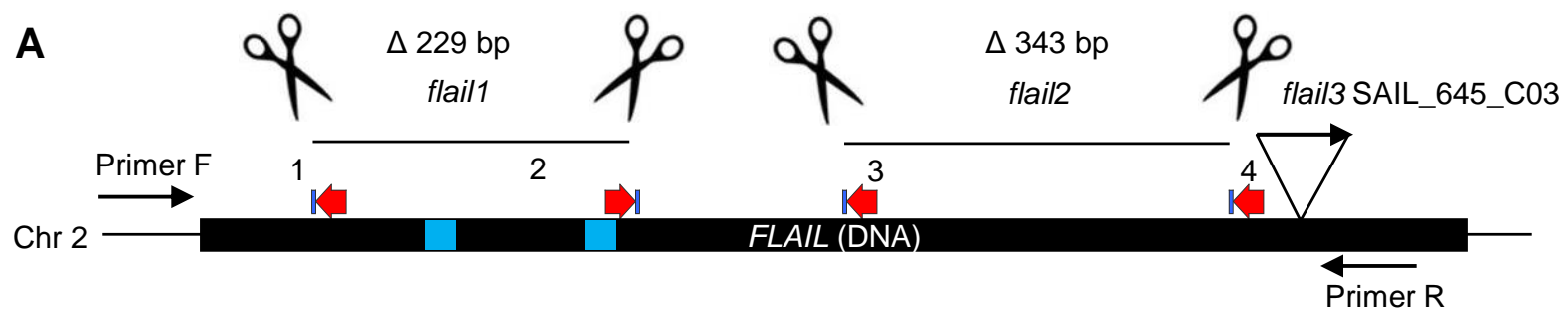

B

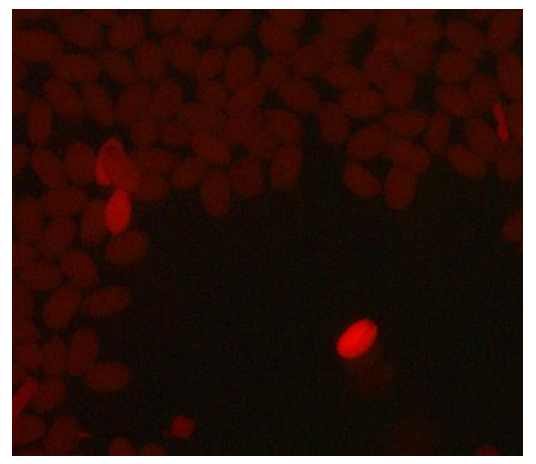

C

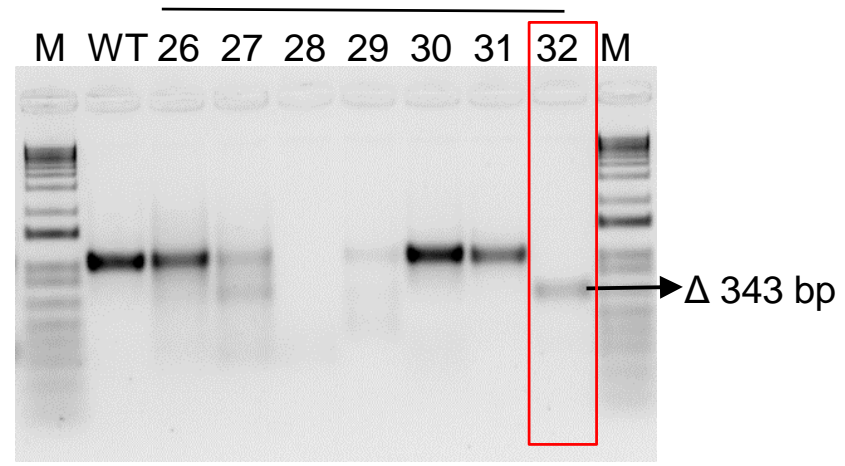

D sgRNA2 protospacer

WT TTTCCAGCAATTACCCTCGTTTGGATCTA...316 bp...CATCCGGTGGAGGTTGTGGAAGATTCACG flail2-32-93 TTTCCAGCAA. $.4343 \mathrm{bp}$ .GAGGTTGTGGAAGATTCACG

E

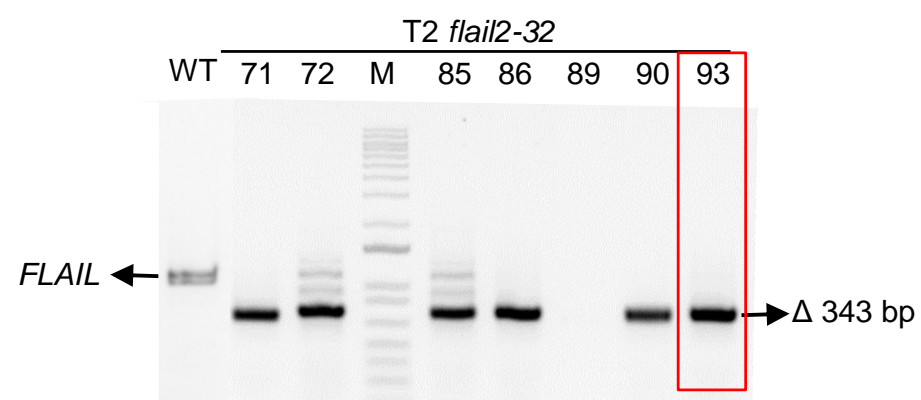

$\mathbf{F}$

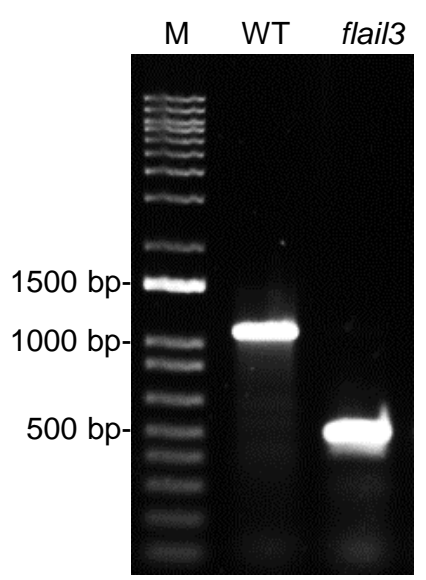




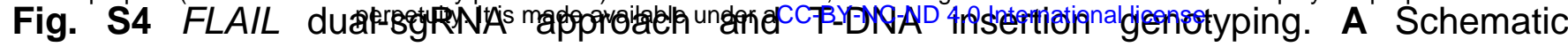

representation of targeted gene FLAIL with locations of two Cas9-directed mutants flail1, flail2, and one T-DNA mutant flail3. Locations of dual-sgRNA target sites were shown in red arrow, primer pair F/R (Mlo2478/2479) were used for PCR testing deletion in flail1 and flail2, triangle indicated the putative position of T-DNA insertion at the FLAIL locus. Blue boxes indicated two sORFs. B Similar to the previous generation of flail1, FLAIL2 construct containing pKIR1.1 enabled red fluorescence selection of flail2 seeds from T1 plants after transformation by floral dipping. C Genotyping of individual T1 plant with red fluorescence, Leaf genomic DNA of T1 plants were PCR amplified. Expected size of PCR product for deletion was $343 \mathrm{bp}$ in flail2 line. flail2-32 in red box was selected for next generation. D In T2 selection, the null mutant flail232-93 without red fluorescence in seeds represented Cas9-free plants, indicated by red box in E. PAM was shown in blue, sgRNA protospacers were in red, and deleted bases were replaced by dots. E PCR analysis of T2 lines. The expected size of the wildtype FLAIL amplicon and the deletion size between Cas9 cut sites in flail2-32-93 were indicated. This inherited Cas9-null segregation line was used for data analysis in Fig. 1. F Genotyping of the T-DNA insertion mutant flail3. Gel sample order (from left to right): lane 1, marker; lane 2, WT; lane 3, flail3, with primer set, Mlo1788 + Mlo1789 + Mlo37; Results clearly showed that flail3 line was homozygous since WT was the only line giving WT band and flail3 line gave the only T-DNA insertion band. 
A

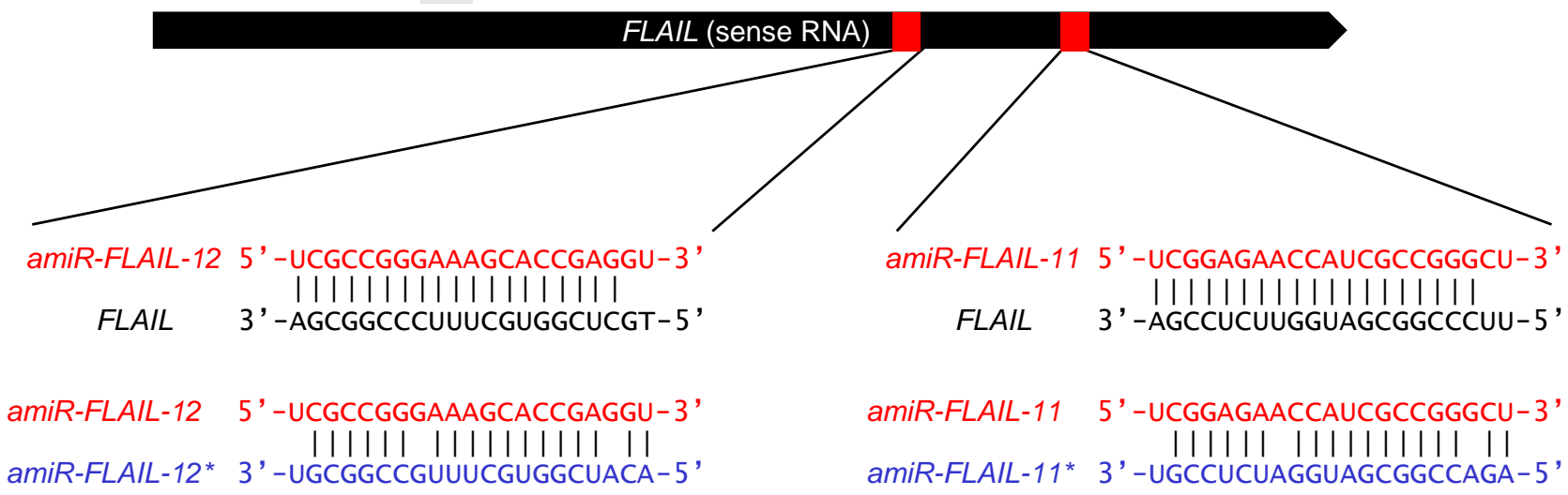

B

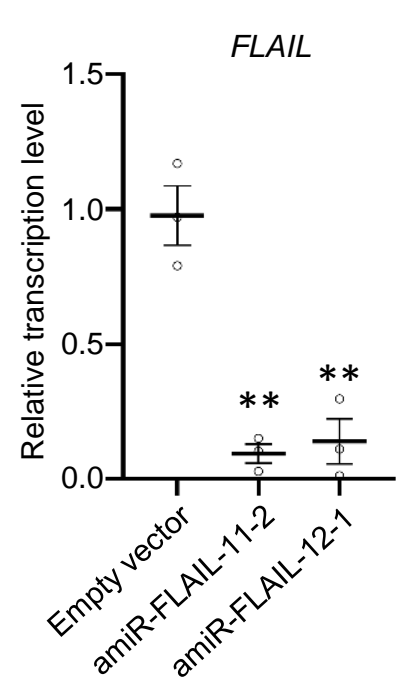

C

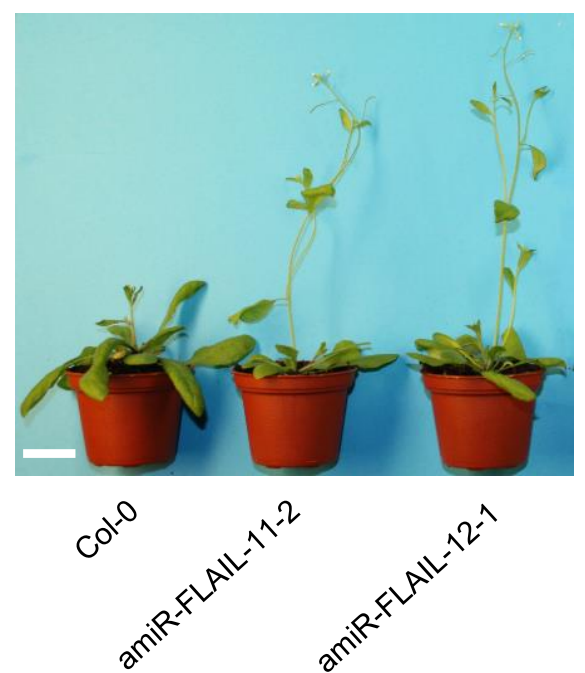

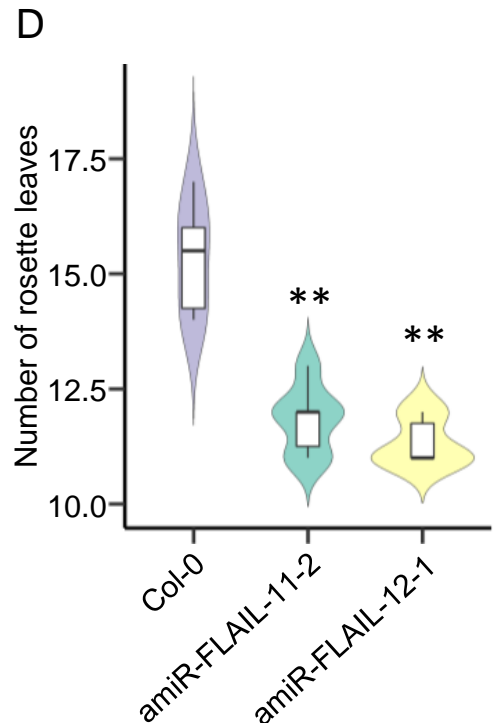

Fig. S5 FLAIL IncRNA functions as a flowering repressor. A Sequences and structures of amiRNA duplexes and the target sites of amiR-FLAILs and amiR-FLAIL ${ }^{*}$. Upper panel, schematic representation of transcribed sense FLAIL RNA. Lower panel, sequences of amiRNA duplexes, amiRNA (in red) target sites, and potential amiRNA* (in blue) on cognate sense FLAIL (in black). B Gene expression of sense FLAIL in amiR-FLAIL plants by RT-qPCR. Transcript levels were normalized to $U B Q$ expression levels. Two representative lines for amiRNA designed transgenic plants were selected. Y-axis showed relative values compared to the expression level of empty vector transformed plants. Grey bars depicted the relative positions of primers used for RT-qPCR analyses. Transcript levels were normalized to $U B Q$ expression levels. Error bars represented s.e.m ( $n=3$ independent 14-day seedling pools). ${ }^{* *}$, $p$ value $<0.01$ analyzed by Student's t-test. C Morphological phenotypes of 4-week-old plants of Col-0 and two amiR-FLAIL plants under a 16-h light/8-h dark growth condition. Scale bar: 2 cm. D Violin graph showed number of rosette leaves after appearance of the first flower bud in Col-0. Data represented the mean of six independent experiments. Boxes spanned the first to third quartile, bold black lines indicated median value for each group and whiskers represented the minimum and maximum values. ${ }^{* *}, p$ value $<0.01$ was indicated by Student's t-test compared to Col-0. 
A

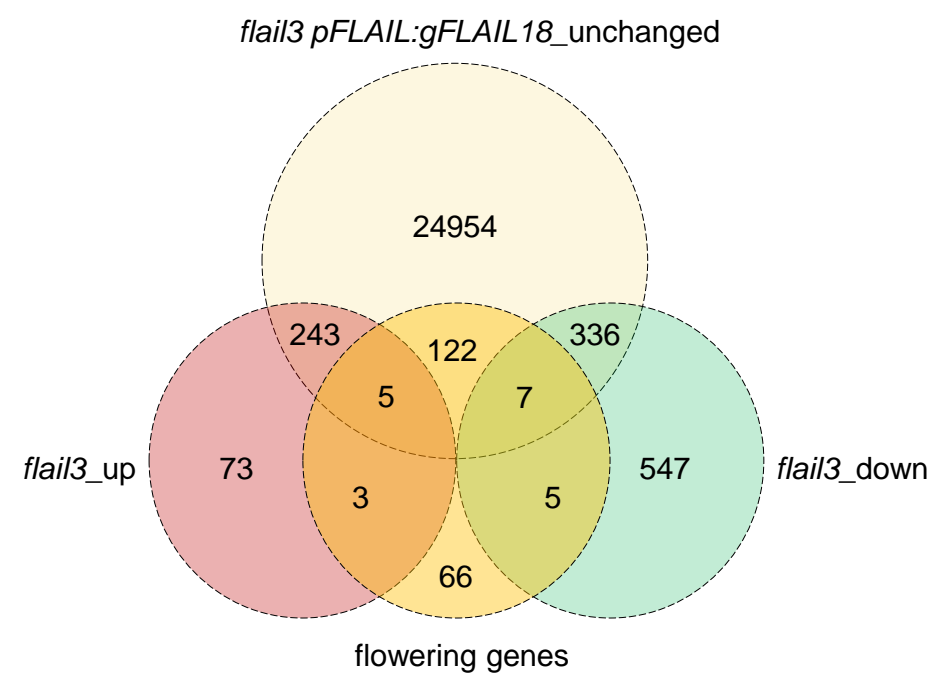

B

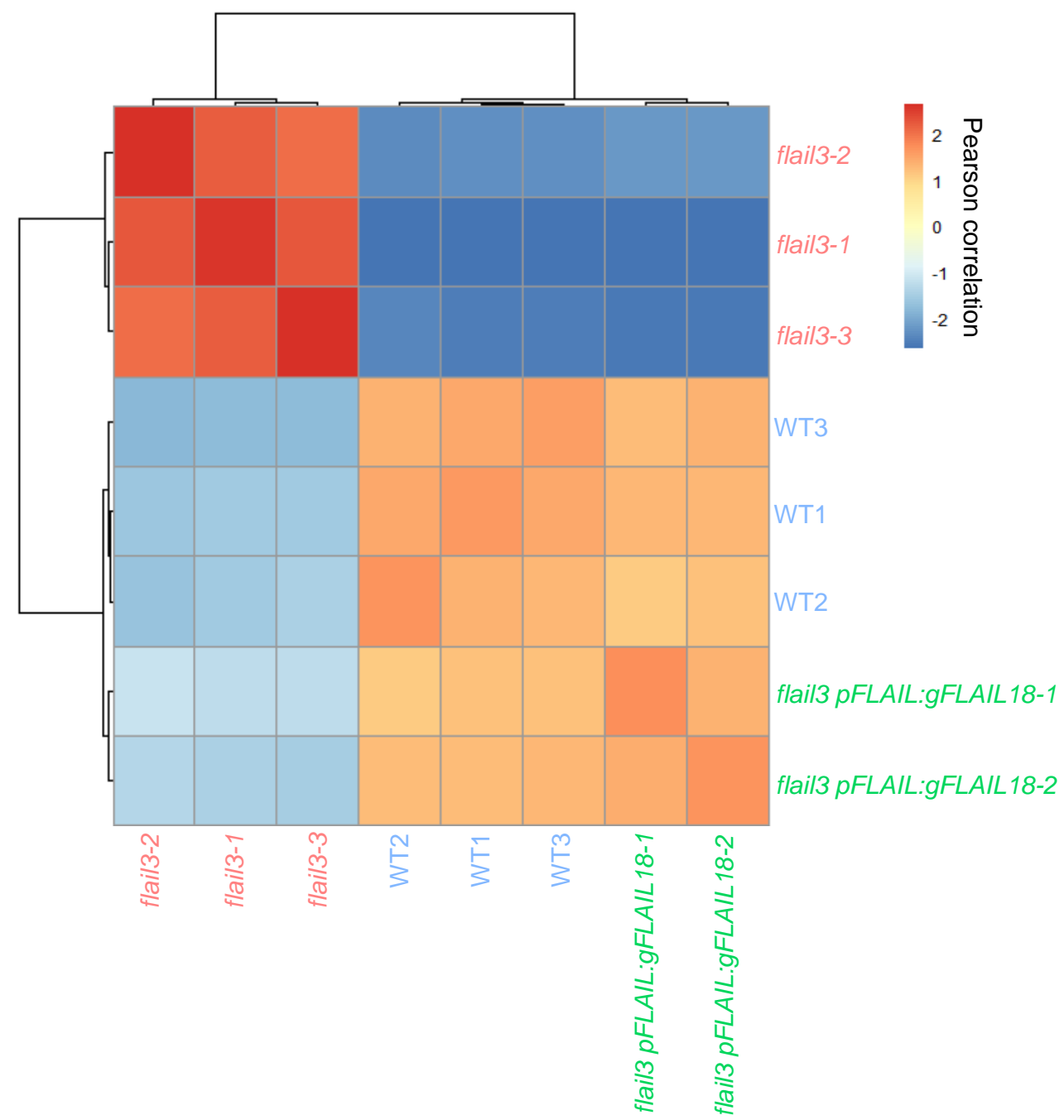

Fig. S6 Genome-wide effects of FLAIL on gene expression by RNA-seq. A Venn diagram of flowering genes overlapped with upregulated and downregulated genes in the flail3 mutant and with unchanged genes in the flail3 pFLAIL:gFLAIL18 complementation plant. B Reproducibility of all genes shown in Fig. 3 from RNA-seq data was demonstrated by clustered heatmap of Pearson correlation coefficients over all independent replicates of RNA-seq in WT, flail3 mutant, and flail3 pFLAIL gFLAIL18 plants. Darker red denoted higher correlation and darker blue represented low reproducibility. 
A

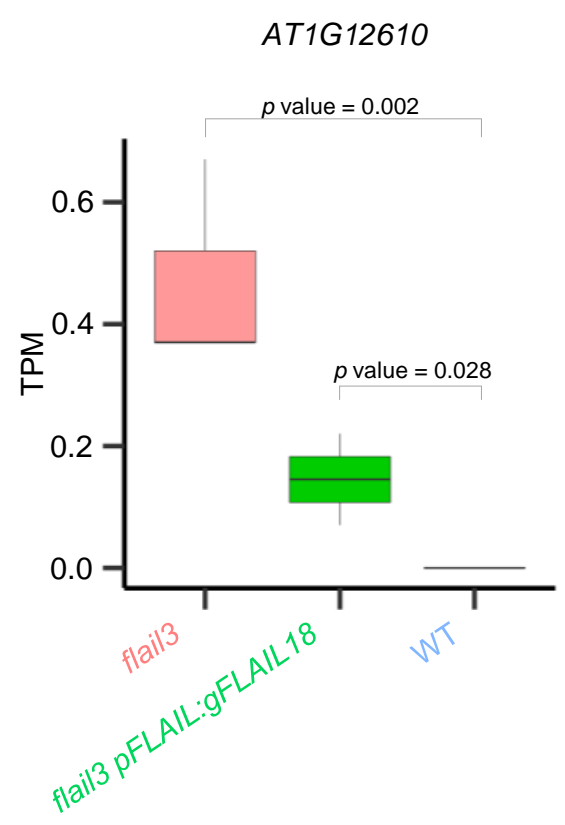

D

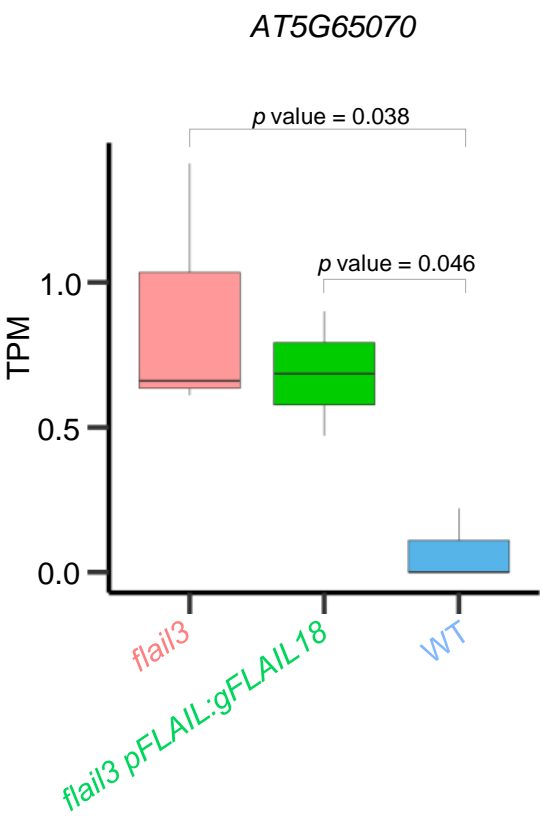

B

AT2G45660

$p$ value $=0.002$

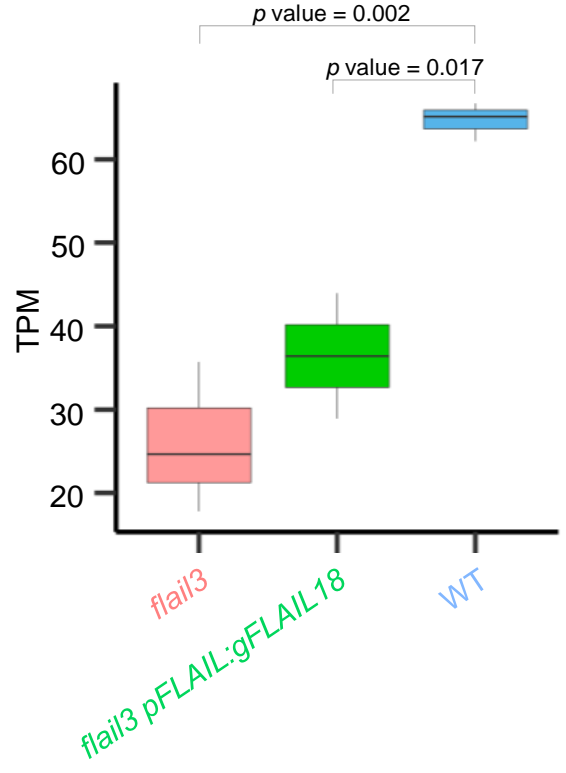

$\mathbf{E}$

AT2G42280

$p$ value $=0.017$

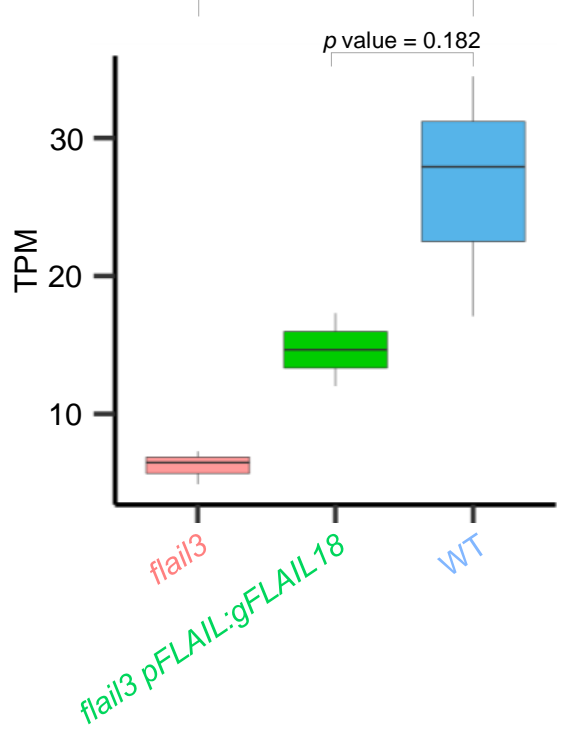

C

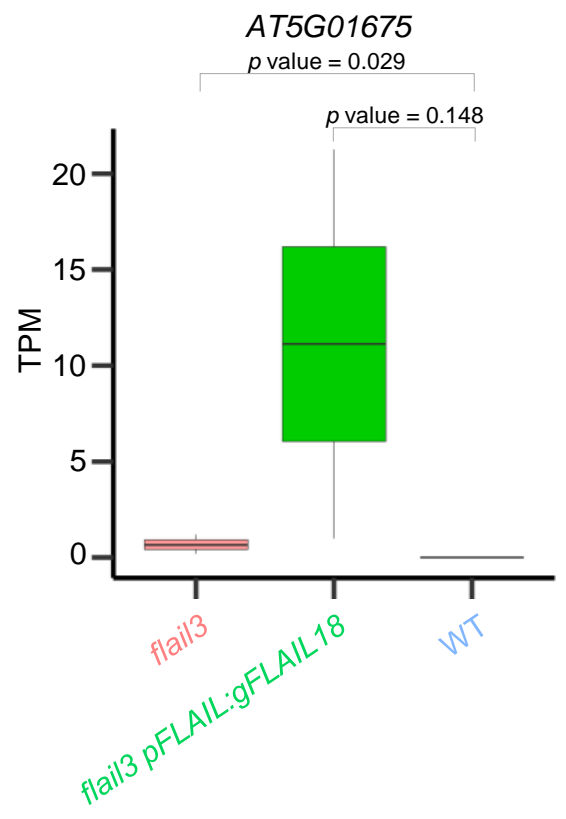

$\mathbf{F}$

FLAIL

$p$ value $=0.007$

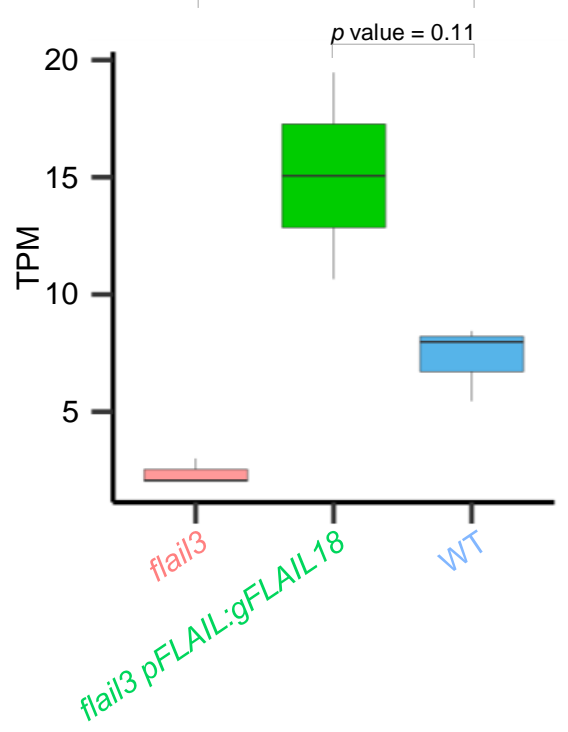

Fig. S7 FLAIL regulates flowering related genes in trans. A-F Genome browser screenshots illustrating the expression of dysregulated flowering genes in flail3 were most fully rescued in complementation line. Normalized read counts (TPM from RNA-seq) were used for differentially expressed flowering genes in WT, flail3, and flail3 pFLAIL:gFLAIL18 plants. Boxes spanned the first to third quartile, bold black lines indicated median value for each group and whiskers represented the minimum and maximum values. $p$ value was denoted by Students' t-test. 
A

Example 1

Odd1/ Odd2 (peak occurred at least one Odd) Even1/ Even2 (peak occurred at least one Even) no peak occurred at least two Lucs $\left\{\begin{array}{l}\text { Luc1/ Luc2/ Luc3 } \\ \text { Luc1/ Luc2/ Luc3 }\end{array}\right.$

Example 2

Odd1/ Odd2 (peak occurred at least one Odd)

Even1 \& Even2 (no peak occurred at both Evens) no peak occurred at least two Lucs $\left\{\begin{array}{l}\text { Luc1/ Luc2/ Luc3 } \\ \text { Luc1/ Luc2/ Luc3 }\end{array}\right.$

Example 3

Odd1 \& Odd2 (no peak occurred at both Odds)

Even1/ Even2 (peak occurred at least one Even) no peak occurred at least two Lucs $\left\{\begin{array}{l}\text { Luc1/ Luc2/ Luc3 } \\ \text { Luc1/ Luc2/ Luc3 }\end{array}\right.$

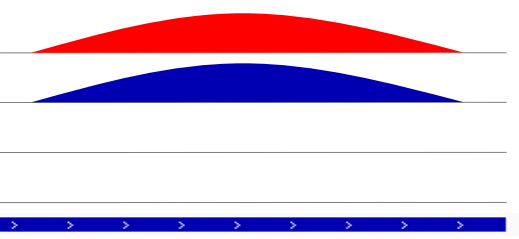

target gene
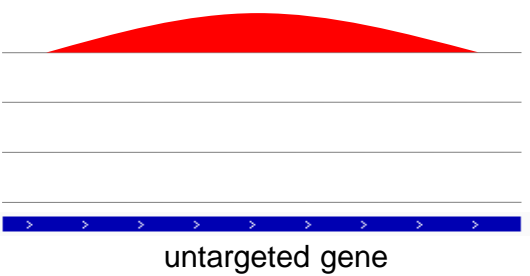

\section{untargeted gene}

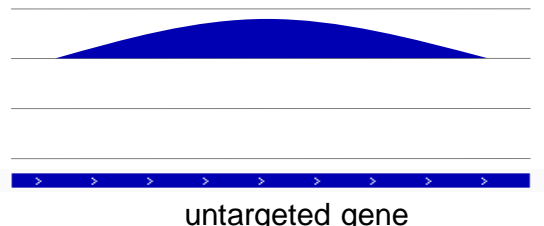

untargeted gene
B
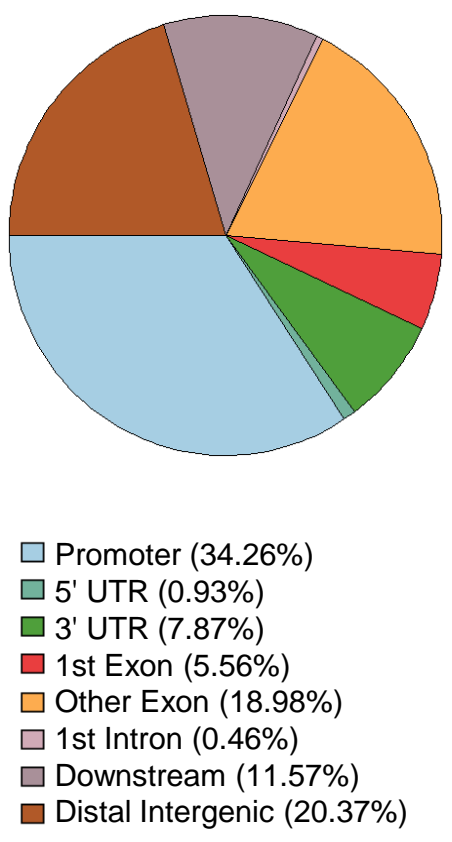

Fig. S8 Strategy to identify genome-wide binding profile of FLAIL analyzed by ChIRP-seq. A Illustration of FLAIL peaks called by CCAT3.0. Only peaks that occurred at target genes from both Even and Odd probed DNA, but not from two or more independent experiments of Luc pools were considered significant enrichment (Example 1), peaks that only occurred from either Even or Odd pools were not considered FLAIL targets (Example 2 \& 3). B A pie chart that was generated by ChIPseeker represented distribution of FLAIL bound regions in the genome. A total of 210 FLAIL bound regions were annotated according to the genomic distribution and FLAIL was enriched predominantly in promoter regions. 
A

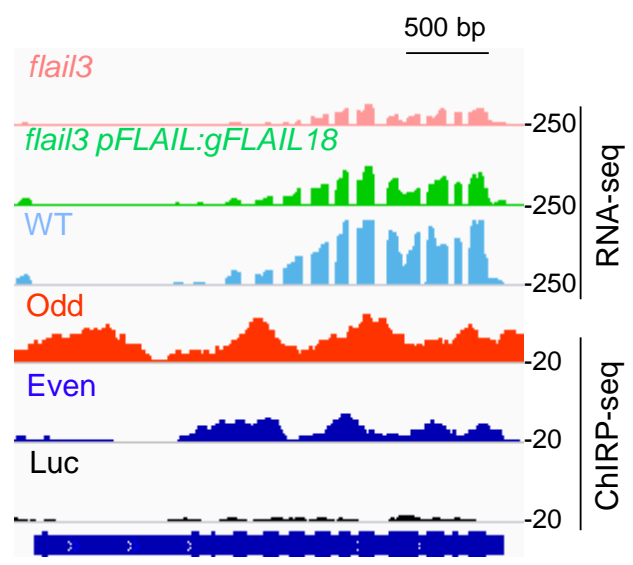

AT1G73600 (NMT3)
B

AT1G73600 (NMT3)

$p$ value $=0.003$

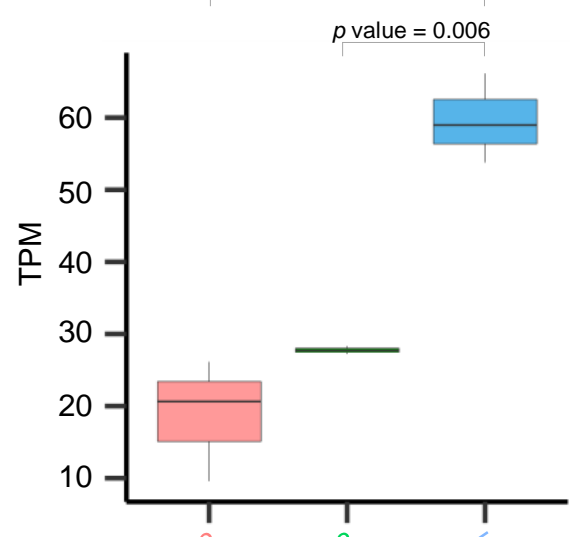

D

\section{AT4G13710 (PLL25)}

$500 \mathrm{bp}$

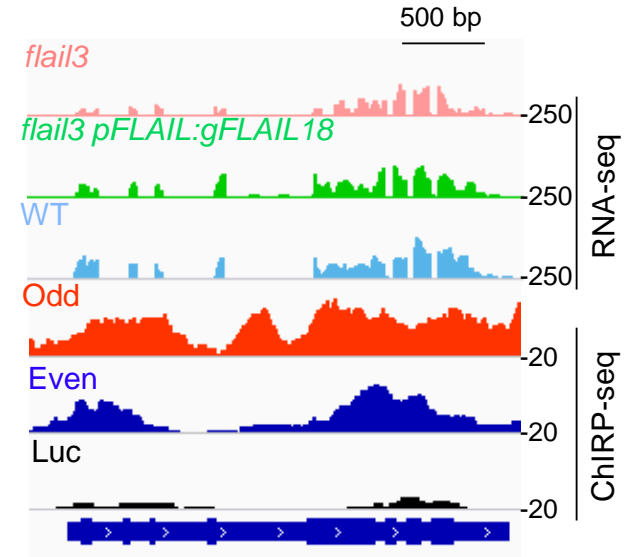

AT4G13710 (PLL25) $p$ value $=0.039$

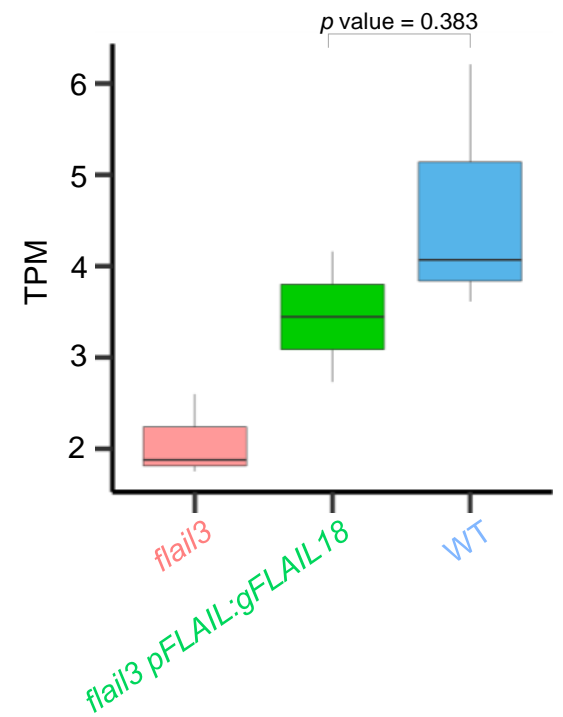

Fig. S9 FLAIL binds chromatin regions of flowering genes for gene regulation. A, C Genome browser screenshots illustrating two more FLAIL bound flowering targets PLL25 and NMT3 in RNA-seq (lane 1-3) and ChIRP-seq (lane 4-6). Their expression that was downregulated in flail3 (lane 1) can be partially or fully rescued in complementation line (lane 2). Both Odd and Even probes identified chromatin binding regions of FLAIL in two more flowering genes NMT3 and PLL25 compared to LUC probes. FLAIL binding peaks were called by CCAT3.0. B, D Normalized read counts (TPM from RNA-seq) for differentially expressed (DE) flowering genes in WT, flail3, and flail3 pFLAIL:gFLAIL18 plants (bottom panel). Boxes spanned the first to third quartile, bold black lines indicated median value for each group and whiskers represented the minimum and maximum values. $p$ value was denoted by Students' t-test. 
A

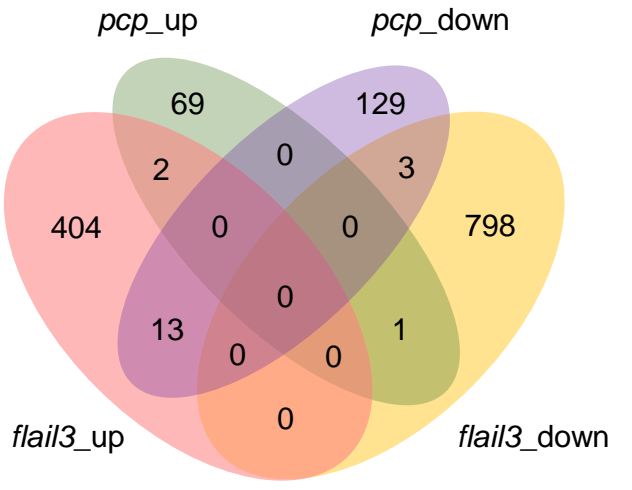

B

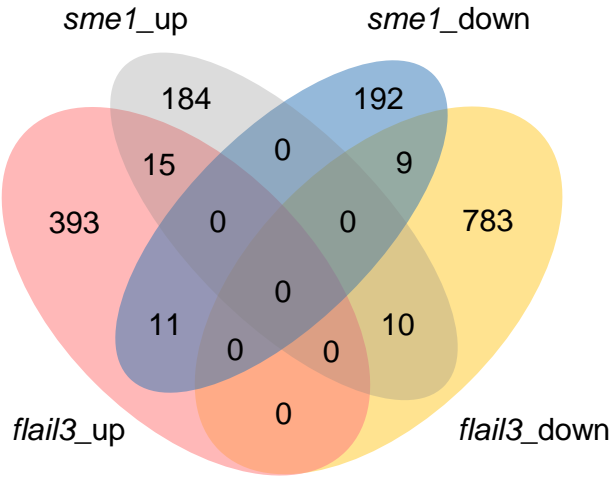

C

flail3

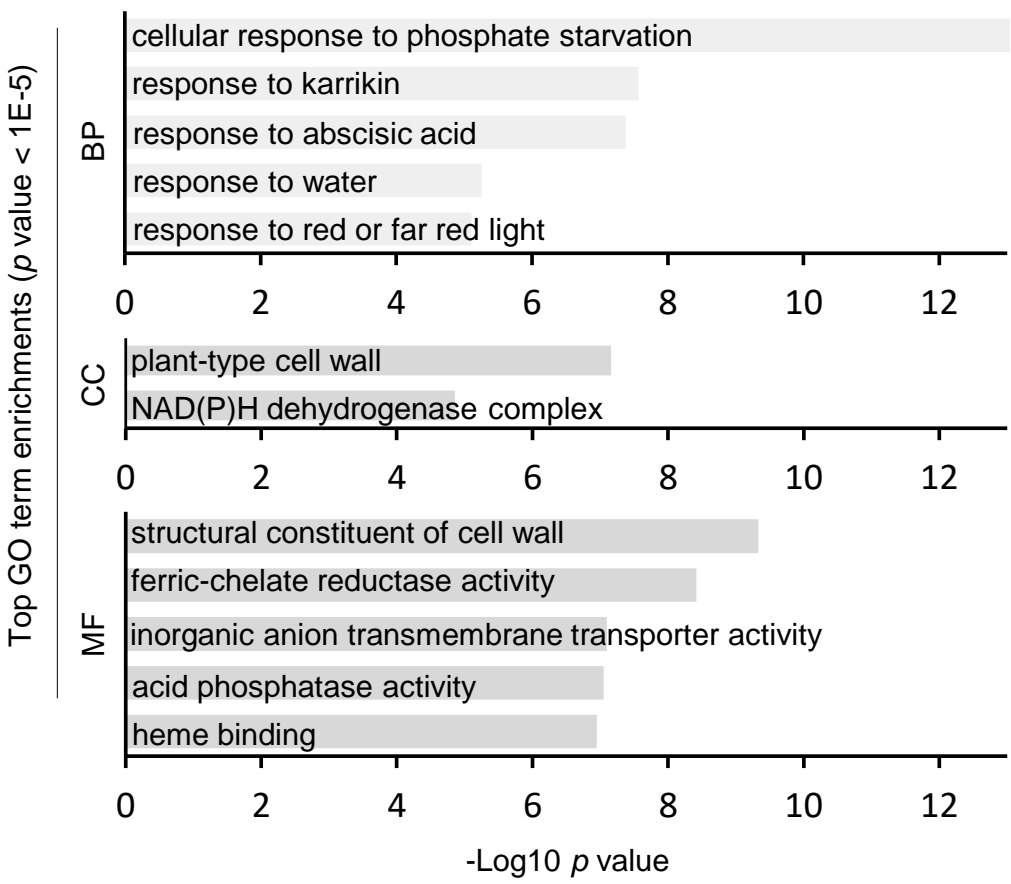

D

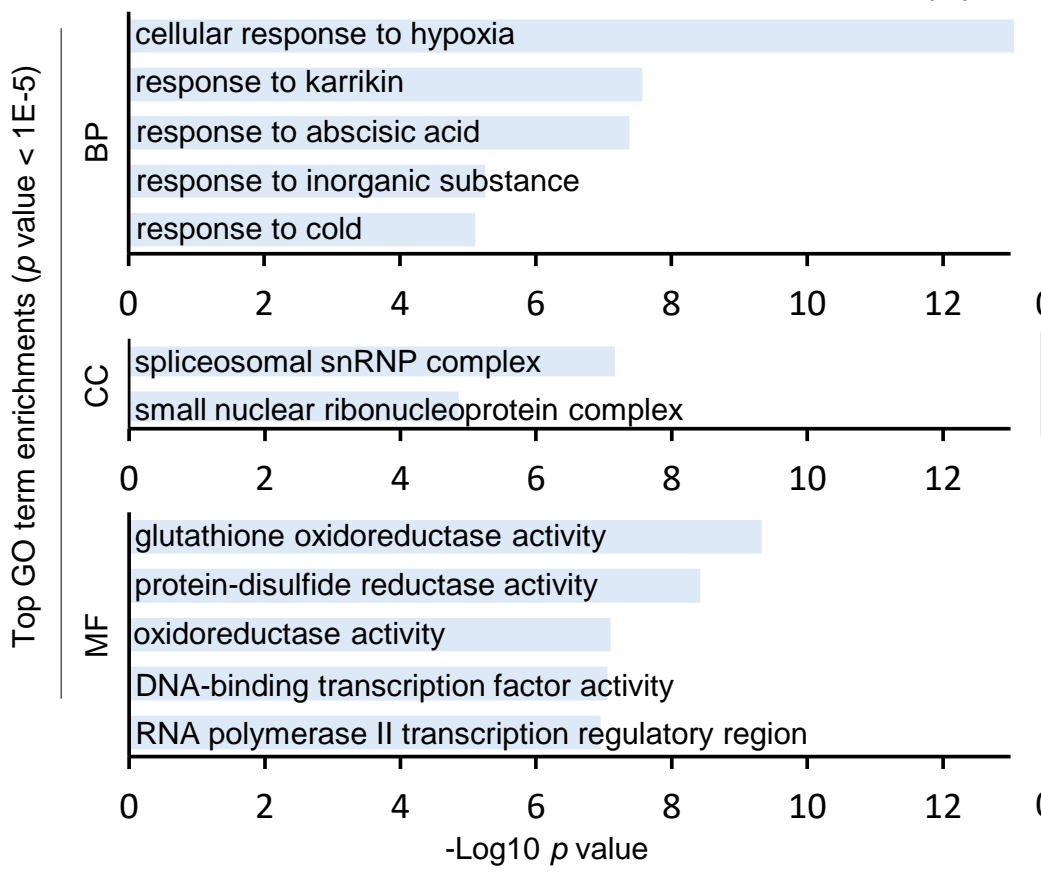

sme1

cellular response to hypoxia response to reactive oxygen species response to heat S-glycoside metabolic process floral meristem determinacy

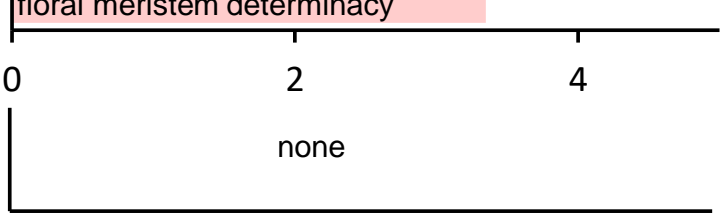

nucleoside transmembrane transporter activity superoxide dismutase activity oxidoreductase activity snRNA binding transferase activity, transferring acyl groups

\begin{tabular}{ll}
\hline 0 & 2 \\
-Log10 $p$ value & 4
\end{tabular}

Fig. S10 FLAIL regulates flowering independent of PCP. A, B Venn diagram showing overlapping differentially expressed genes (up/down) sets between flail3 and $p c p$ (also called sme1) mutants. C, D GO analysis of the DEGs in RNA-seq data of flail3 and pcp /sme1 mutants. Y-axis indicated the GO categories including biological process (BP), cellular component (CC) and molecular function (MF); X-axis showed -Log10 $p$ value with the cutoff 0.05 . Highly enriched GO terms of dysregulated mRNAs analyzed by the Metascape with 5 top enrichment scores. 